\title{
Pathogenesis of human cytomegalovirus in the immunocompromised host
}

\author{
Paul Griffiths (10) ${ }^{\bowtie}$ and Matthew Reeves (1)
}

Abstract | Human cytomegalovirus (HCMV) is a herpesvirus that infects $\sim 60 \%$ of adults in developed countries and more than $90 \%$ in developing countries. Usually, it is controlled by a vigorous immune response so that infections are asymptomatic or symptoms are mild. However, if the immune system is compromised, $\mathrm{HCMV}$ can replicate to high levels and cause serious end organ disease. Substantial progress is being made in understanding the natural history and pathogenesis of HCMV infection and disease in the immunocompromised host. Serial measures of viral load defined the dynamics of HCMV replication and are now used routinely to allow intervention with antiviral drugs in individual patients. They are also used as pharmacodynamic read-outs to evaluate prototype vaccines that may protect against HCMV replication and to define immune correlates of this protection. This novel information is informing the design of randomized controlled trials of new antiviral drugs and vaccines currently under evaluation. In this Review, we discuss immune responses to HCMV and countermeasures deployed by the virus, the establishment of latency and reactivation from it, exogenous reinfection with additional strains, pathogenesis, development of end organ disease, indirect effects of infection, immune correlates of control of replication, current treatment strategies and the evaluation of novel vaccine candidates.

Infectious mononucleosis A syndrome of fever and malaise with an excess of lymphocytes in the blood, many of them atypical in form.
Human cytomegalovirus (HCMV; also known as human herpesvirus 5) is the prototype member of the Betaherpesvirinae. Like all herpesviruses, it establishes latency and persists for the life of the individual. Infection with HCMV is common throughout the globe ${ }^{1}$. The proportion of adults with specific IgG antibodies approximates to $60 \%$ in developed countries and more than $90 \%$ in many developing countries ${ }^{1}$. Infection is more common in those from lower socio-economic groups and from non-Caucasian backgrounds ${ }^{2}$. Children born in the UK to women who have moved from high-risk countries have the lowered risk of their adopted country ${ }^{2,3}$. The saliva and urine of young children are major sources of virus, especially for those with childcaring responsibilities $^{4}$. HCMV is not highly contagious, with a basic reproductive number of $\sim 1.7-2.4\left(\mathrm{REFS}^{5-7}\right)$. It can also be spread sexually, by transfusion of whole blood or by organ transplantation ${ }^{8,9}$. It is important to note that there are usually no symptoms associated with HCMV infection, except for occasional cases of infectious mononucleosis. This is because a robust immune response to HCMV normally prevents the high viral loads required to cause the end organ disease (EOD) seen in immunocompromised individuals. However, despite the absence of overt symptoms, there is evidence that infected individuals may have long-term adverse outcomes related to induction of a chronic inflammatory cell-mediated immune response to this apparently innocuous virus (indirect effects) ${ }^{10}$.

The natural history of HCMV infection is complex, with three different subtypes of infection ${ }^{9}$. Primary infection occurs when an individual with no immunity against this virus becomes infected for the first time. Afterwards, the virus establishes latency from which it may reactivate (second type of infection). The third type of infection is called reinfection when contact with an infectious individual results in superinfection of someone who has already been infected, despite their possession of natural immunity ${ }^{9}$. Any of these three subtypes of infection can complicate pregnancy, making HCMV the commonest cause of congenital infection ${ }^{11}$. It is also the most common and the most serious opportunistic infection after solid organ transplantation (SOT) or haematopoietic stem cell transplantation (SCT) and remains an important opportunistic infection in individuals with $\mathrm{HIV}^{9,12,13}$.

In this Review, we will focus on HCMV infection in immunocompromised individuals. We aim to integrate 
information about serial measures of the viral load to explain features of pathogenesis and differences between distinct patient groups, show how active infection (although asymptomatic) is routinely monitored in selected patients and illustrate how prototype vaccines can be evaluated for efficacy. We will emphasize the evidence provided by double-blind, placebo-controlled randomized clinical trials (RCTs) of active or passive immunotherapy specific for HCMV.

\section{HCMV immune evasion and viral latency}

Our understanding of the complex interaction of HCMV with the immune response has been informed, in part, by comparative analyses of established laboratory-adapted

\section{Box 1 | Human cytomegalovirus strains and their genomes}

Human cytomegalovirus (HCMV) was first grown in 1957 using the then new technology of cell culture ${ }^{127}$. It was difficult to propagate serially, but laboratory-adapted strains such as Ad169 (cultured from adenoids) and Towne (named after a patient) released more cell-free virus into the extracellular fluid and were shared widely among researchers. The Nobel laureate and co-discoverer of HCMV, Thomas Weller, anticipated the possibility that this laboratory adaptation may have selected for genetic changes ${ }^{128,129}$. In 1990, this was confirmed when it was reported that a $20-\mathrm{kb}$ genomic segment was missing from some strains (designated the ULb' region) ${ }^{130}$. The 19 genes encoded within ULb' were clearly dispensable for growth in fibroblast cell culture. Thus, their retention in wild-type strains argues that they have crucial roles during infection in vivo.

One aspect found to be associated with the ULb' region was viral tropism and entry. A trimer of proteins (glycoprotein $\mathrm{H}(\mathrm{gH}), \mathrm{gL}$ and $\mathrm{gO}$ ) form a complex on the surface of the virion that binds to platelet-derived growth factor receptor- $\alpha$ (PDGFR $\alpha$ ) and co-receptors on the surface of fibroblasts ${ }^{131}$. Entry into the fibroblasts then occurs via fusion at the plasma membrane at neutral $\mathrm{pH}^{132}$ (FIG. 1). By contrast, $\mathrm{gH}$ and $\mathrm{gL}$ can also bind to three distinct small proteins (encoded within the ULb' region) to form a pentameric complex ${ }^{133}$. This complex binds to numerous receptors including CD147 and neuropilin 2 receptor on epithelial and endothelial cells, facilitating entry through a pH-dependent endocytic pathway ${ }^{118,134,135}$ (FIG. 1). What has become clear from the ability to reconstruct the wild-type $\mathrm{HCMV}$ genome using bacterial artificial chromosome technology is that HCMV strains that express the pentameric complex propagate in a highly cell-cell fashion, but when the pentamer is absent (as in Ad169 or Towne laboratory strains), a high proportion of cell-free virus is produced ${ }^{136}$.

We now know (through whole-genome sequencing) that HCMV has the largest genome of any virus known to infect humans (double-stranded DNA of 235-250kb) ${ }^{137-139}$. HCMV encodes $\sim 170$ canonical open reading frames although non-canonical open reading frames may increase this coding capacity fivefold ${ }^{140,141}$. Despite the size of the genome, it is notable that as much as $70 \%$ of the viral genome (including the example of $\mathrm{ULb}^{\prime}$ ) is dispensable for growth in vitro ${ }^{142}$. Although it is understandable that the early pioneering researchers utilized the most tractable strains to work with in vitro, scientific experimentation remains a trade-off between authenticity (are we measuring a laboratory phenomenon?) and tractability. What is now clear is that many of the genes encoded within ULb' are involved in cell tropism and immune evasion, explaining why they became dispensable in vitro. Indeed, $\mathrm{HCMV}$ encodes more genes for immune evasion than it does to produce the virion itself.

Genetic differences in strains of HCMV need to be borne in mind when considering whether particular vaccines can protect against primary infection, reinfection or reactivation. At present, information from clinical trials is only available for $\mathrm{gB}$. The gB-MF59 vaccine is based on the Towne strain that has gB1 (out of four possible genotypes ${ }^{143}$ ). There is some evidence that women immunized with gB-MF59 had better protection against primary infection with natural strains bearing $\mathrm{gB} 1$ than against viruses with other $\mathrm{gB}$ genotypes ${ }^{144}$. The plasmid vaccine ${ }^{113,145}$ is based on Ad169 (which has $\mathrm{gB} 2$ ), as are the Hookipa Pharma ${ }^{114}$ and Merck ${ }^{115}$ vaccines.

In addition to mutations selected in vitro (such as the UL/b' example), variation occurs naturally in vivo. Whole-genome sequencing of clinical samples is starting to describe the level of variation in terms of genotypes of numbered genes and document that not all circulating strains encode exactly the same repertoire of genes ${ }^{146-148}$. The extensive variation seen has the potential to affect virus pathogenesis in vivo, but no definitive studies have yet been reported. strains of HCMV (BOX 1) and clinical isolates. An overview of infection and establishment of latency is shown in FIG. 1. Variation in sequence occurs naturally between HCMV strains and has the potential to impact pathogenesis and vaccine development. Note that individual genes of HCMV are numbered sequentially with the unique long, unique short or terminal repeat regions of the genome: thus, UL54 refers to the 54th gene in the unique long region.

Numerous studies have demonstrated that HCMV encodes countermeasures against a spectrum of immune responses $^{14-16}$ (FIG. 2a). This arsenal of immunomodulatory functions is likely a reflection of the natural history of the virus, providing the capacity to establish lifelong infections of the host as well as to reinfect people with an existing infection despite the presence of a substantial immune response - particularly an enlarged T cell compartment dominated by anti-HCMV T cell responses that often exceed $10 \%{ }^{17}$. The complexity of these immunological interactions has been reviewed extensively elsewhere ${ }^{18-25}$. Suffice it to say that HCMV-encoded gene functions target antigen presentation by major histocompatibility complex (MHC) class I and class II molecules, utilize cytokine mimicry to exert paracrine functions against immune cells and encode proteins that antagonize the range of innate immune responses directed against the virus (FIG. 2a). Despite this, HCMV infection or reactivation in the immunocompetent individual is rarely a cause of morbidity, implying that the surfeit of immune evasion mechanisms encoded by HCMV is imperative for long-term persistence in the host but not sufficient to completely evade immunosurveillance.

It is tempting to propose that the long-term solution for HCMV to evade immune responses is by 'hiding' from them, rather than 'running' from them, as seen in HIV infection ${ }^{26}$. This strategy of hiding from immunosurveillance is exemplified by the virus spreading cell to cell within a sanctuary site of persistence. This is coupled with an ability to establish latency, providing a mechanism by which the virus can go to ground if the immune system gains the upper hand, only to return later through reactivation if the immune system becomes impaired.

The ability to establish lifelong latent infections of the host is a defining characteristic of herpesvirus infections ${ }^{27}$. An overview of HCMV latency and reactivation is shown in FIG. 2b. HCMV establishes latent infections in bone marrow haematopoietic progenitor cells ${ }^{28}$. Additionally, tissue endothelial cells may be another reservoir of latent or persistent infection and, thus, may contribute to HCMV pathogenesis in organ transplantation ${ }^{29}$. Latency can be defined as the persistence of the viral genome in an absence of lytic replication and virus production coupled with the retention of a capacity to reactivate when specific conditions are met. Typically, cellular differentiation and inflammation-associated signalling appear to be important events required for reactivation in the myeloid lineage $\mathrm{e}^{30-35}$. Specifically, differentiation of myeloid progenitors to macrophages or dendritic cells drives reactivation via the induction of a key viral gene locus required for lytic infection - the major immediate early (MIE) region ${ }^{28}$. 
Owl's eye inclusion bodies Intranuclear inclusions seen in histopathological sections from organ biopsies with human cytomegalovirus (HCMV) infection.

Pneumonitis

Inflammation in the interstitial tissue of the lung rather than the airways
The activity of inflammatory cytokines as inducers of MIE gene expression and HCMV reactivation (for example, tumour necrosis factor (TNF) and interleukin-6 (IL-6)) may be important in the process of organ transplantation which is associated with substantial inflammation ${ }^{36-38}$ (FIG. 2b). Indeed, one of the first reports of reactivation of naturally latent $\mathrm{HCMV}$ in vitro was achieved using the cocktail of cytokines released from allogeneically stimulated $\mathrm{T}$ cells ${ }^{30}$. Furthermore, sepsis increases the incidence of HCMV reactivation in immunocompetent patients in intensive care, which could be linked with the inflammation associated with bacterial infection complications ${ }^{39,40}$. Consequently, it is hypothesized that inflammation-associated signalling is a key driver of reactivation. The fact that HCMV has evolved mechanisms to modulate host cell signalling during latency and reactivation would be consistent with this.

A common refrain is that immunosuppression is a key trigger of HCMV reactivation yet HCMV reactivation can be modelled in vitro in cell culture systems. Thus, a distinction between clinical reactivation and cellular reactivation is important. Clinical reactivation is the detection of viraemia in individuals who are seropositive. Cellular reactivation is the re-initiation of viral replication in differentiated permissive cells. Importantly, understanding the relationship between these two events is likely crucial for understanding the basis of viraemia in immunocompromised individuals seropositive for HCMV.

Studies of cells isolated from healthy immunocompetent individuals have provided evidence of HCMV lytic gene expression in dendritic cells (cellular sites of HCMV reactivation) despite no evidence of viraemia. This observation suggests that HCMV latency and reactivation are an ongoing event in the host - an event that is controlled by the prodigious immune response directed against it. Indeed, this constant exposure of HCMV to the immune system likely explains the immunological space devoted to control of the virus. Thus, in individuals with compromised immune systems (for example, after organ transplantation), reactivation is still occurring but the loss of immune function allows the virus to replicate unchecked, leading to viraemia and, ultimately, disease. Importantly, in many of these scenarios where we observe immunosuppression there is a concomitant inflammatory state driven by co-infections or allogeneic $\mathrm{T}$ cell responses that exacerbate the situation due to the associated inflammation having proviral roles in replication.

It is noteworthy that HCMV is often one of the first viral pathogens to be identified diagnostically in patients with transplantation. This pre-eminent emergence is easily explained if we assume the virus is being controlled at the point of reactivation rather than at latency. Essentially, immunosuppression is akin to releasing the brake rather than representing the trigger. What remains to be understood is the relative contributions that different aspects of the innate and adaptive immune responses make towards the control of HCMV infection in vivo in different clinical settings of immunosuppression or immunodeficiency. The first clues will come from studies of immune function in different patient cohorts and, specifically, identifying the loss of which elements of the response are responsible for pathogenesis (FIG. 2b).

\section{Natural history and pathogenesis}

The natural history of HCMV consists of frequent infection that sometimes leads to viraemia. Only some of those with viraemia then proceed to develop EOD. In this section, we discuss each immunocompromised patient group in turn, each of which have different reasons for being immunocompromised (BOX 2). Notably, each EOD is similar across different patient groups (for example, HCMV retinitis has the same clinical features in all patient groups). Diagnosis of HCMV EOD requires evidence of symptoms at the affected site together with a biopsy showing histopathological changes of owl's eye inclusion bodies and/or immunocytological staining to demonstrate productively infected cells, except in the case of retinitis where the characteristic clinical appearance of haemorrhage in retinal vessels accompanied by exudate is accepted ${ }^{41}$.

Retinitis may be reported by the patient as a visual loss or 'floaters' passing across the visual field, or may be asymptomatic and identified by clinical examination alone. HCMV retinitis is attributed to lytic infection, a conclusion supported by clinical resolution with antiviral therapy (systemic ganciclovir plus intravitreal foscarnet if the retinitis is immediately sight-threatening). Healing is by fibrosis, predisposing the patient to future retinal detachment, a cause of major vision loss. When antiretroviral therapy was introduced, some individuals with HIV developed immune recovery uveitis, an inflammatory response to the presence of HCMV antigens within the eye $\mathrm{e}^{42}$. This condition may cause more visual disturbance to a patient than the underlying retinitis.

Pneumonitis is typically seen soon after SCT. The viral load in bronchoalveolar fluid is high ${ }^{43}$. The pathology involves interstitial recruitment of lymphocytes increasing the distance that oxygen has to pass between the alveoli and blood vessels in the lung. There is evidence for an immunopathological contribution to this disease $\mathrm{e}^{44}$.

The remaining EODs of hepatitis, gastrointestinal ulceration and rarer conditions such as nephritis or pancreatitis are assumed to be caused by lytic infection.

End organ disease in solid organ transplantation. The very first cases of SOT were complicated by HCMV pneumonitis, with high mortality ${ }^{45}$. The pioneers in organ transplantation mitigated this by lowering doses of immunosuppressive drugs, but at the risk of allowing graft rejection to occur. Balancing the need for immunosuppression (graft rejection) with a need for immune control (HCMV) benefited greatly from the development of less toxic immunosuppressive drugs coupled with the HCMV antiviral ganciclovir and its prodrug valganciclovir. However, the problem of HCMV EOD still persisted. Cases of HCMV pneumonitis continued to occur without prior warning; although many patients had fever, this sign is so common post transplant that it is not a specific indicator of HCMV infection. HCMV was also detected histopathologically in biopsies from other organs beyond the lungs, particularly the gastrointestinal 

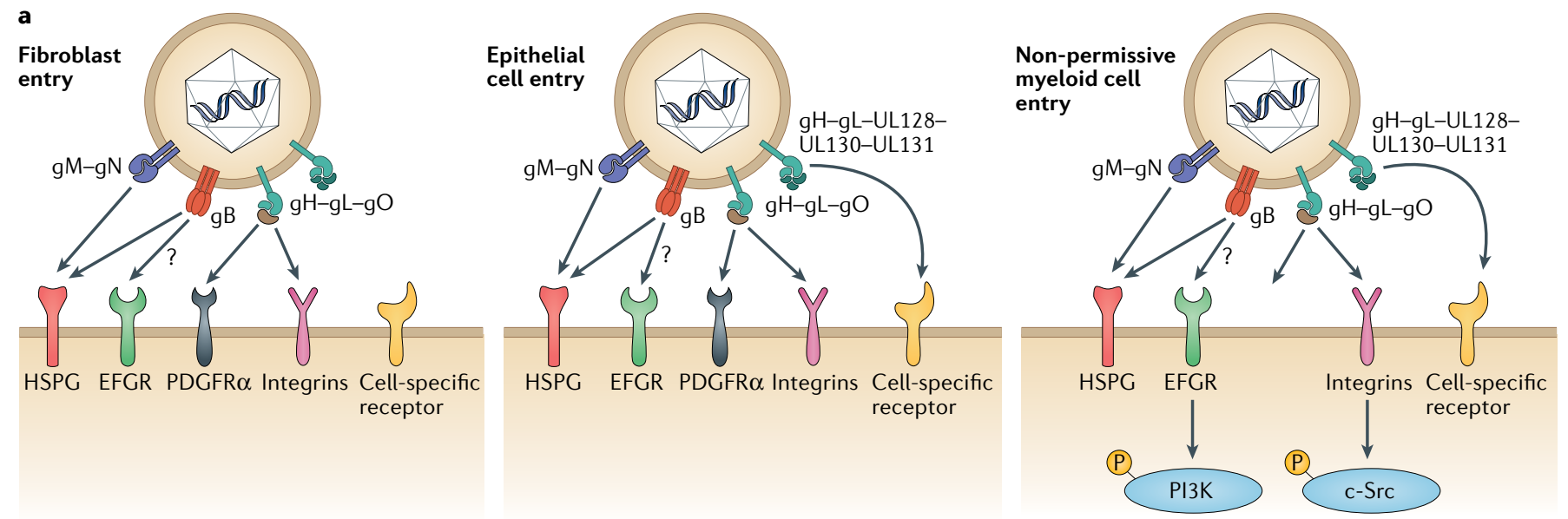

$\mathrm{pH}$-independent fusion (fibroblasts)

Endocytosis (epithelial and endothelial cells)
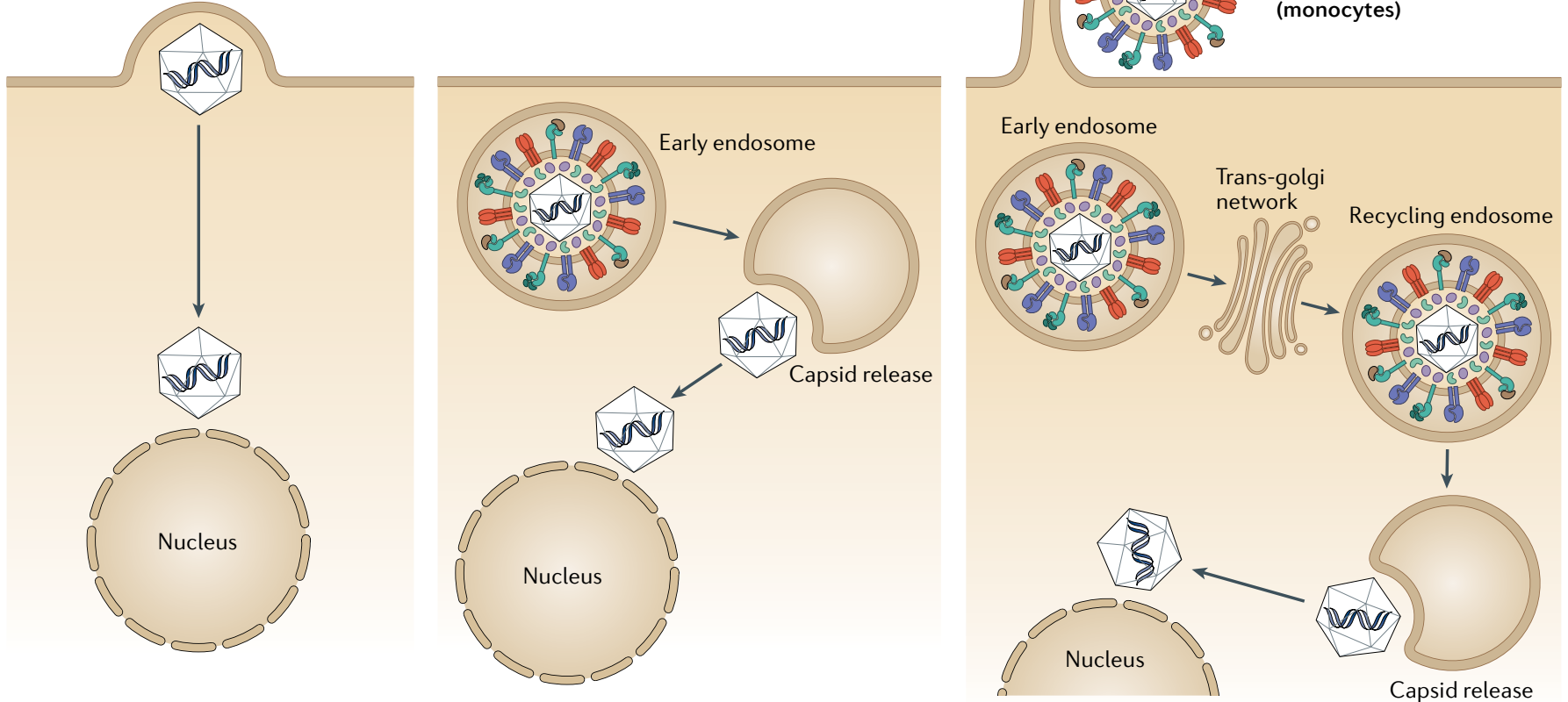

b
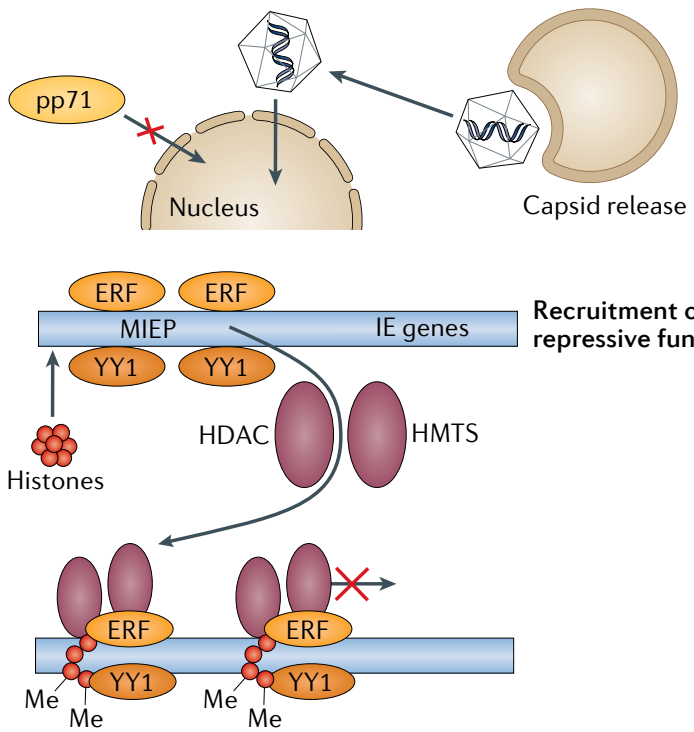

Transcriptional repression
Recruitment of repressive functions

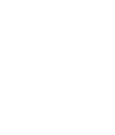

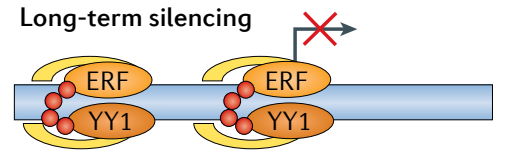

$+1$

Long-term transcriptional c
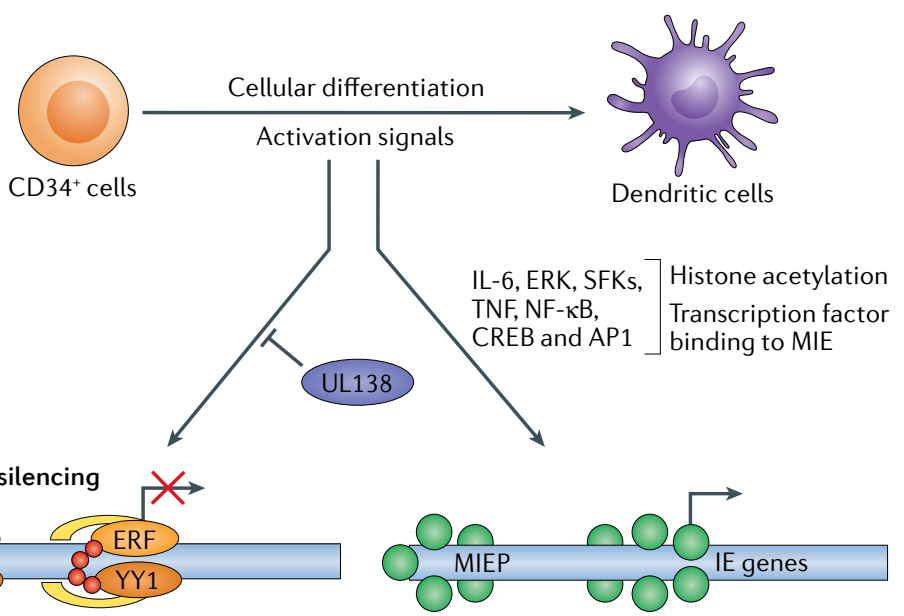
repression

Transcriptional activation 
4 Fig. 1 | Overview of human cytomegalovirus entry into target cells and the establishment of latency in non-permissive myeloid cells. a | Human cytomegalovirus (HCMV) glycoproteins on the surface of the virion engage with receptors on the surface of cells and can drive entry by multiple processes in a cell type-dependent manner. In fibroblasts, glycoprotein $\mathrm{H}(\mathrm{gH}), \mathrm{gL}$ and $\mathrm{gO}$ form a trimer that binds to platelet-derived growth factor- $\alpha$ (PDGFR $\alpha$ ) and co-receptors. This binding triggers $\mathrm{gB}$ to fuse directly with the plasma membrane at neutral $\mathrm{pH}$. In permissive epithelial and endothelial cells, $\mathrm{gH}$ and $\mathrm{gL}$ form a pentameric complex with three other proteins encoded within the ULb' region (UL128-UL130-UL131). This pentameric complex binds to neuropilin 2 and triggers $\mathrm{pH}$-dependent endocytosis. The fusion activity of $\mathrm{gB}$ becomes relevant for escape from the endosome. For both cell types, once the capsid and associated tegument proteins are released into the cytoplasm they move independently to the nucleus, where virion DNA interacts with the nuclear pore complex to transition into the nucleus. Infection of myeloid cells (including potential sites of latency) involves macropinocytosis. In myeloid cells where HCMV establishes latency (that is, CD34 ${ }^{+}$ cells), activation of epidermal growth factor receptor (EGFR) and integrin-mediated src family kinase (SFK) signalling via $\mathrm{gB}$ and pentamer, respectively, is required for the trafficking of HCMV DNA contained within the capsid to the nucleus via recycling endosomes. An overview of cellular receptors is provided in REF. ${ }^{118} . \mathbf{b}$ | Establishment of latency is dependent on effective silencing of major immediate early (MIE) gene expression. In CD34+ cells, this is likely a combination of host and viral-encoded events including a failure of virion transactivators (for example, pp71) to enter the nucleus coupled with a host environment of high levels of transcriptional repressors of the MIE promoter (MIEP). The result is establishment of a repressive chromatin phenotype driving MIEP silencing that is maintained by viral UL138 gene expression. $\mathbf{c} \mid$ Cellular differentiation to a dendritic cell promotes induction of transcription from the MIE locus through the activity of host chromatin remodelling enzymes. This process is responsive to inflammatory cytokine signalling (for example, tumour necrosis factor (TNF) and interleukin 6 (IL-6)) through extracellular signal-regulated kinase (ERK) and SFK signalling pathways. Binding sites for multiple transcription factors (for example, nuclear factor- $\kappa \mathrm{B}(\mathrm{NF}-\kappa \mathrm{B})$, cAMP response element binding protein (CREB), activator protein 1 (AP1)) in the MIEP have been hypothesized to be important for the control of MIE gene expression upon reactivation. Part $\mathbf{a}$ is adapted from Murray et al. ${ }^{119}, 2018$ CC BY 4.0 (https://www.mdpi.com/2076-0817/7/1/30).

tract, in patients with oesophagitis, gastritis or colitis. It was also recognized in the eye when patients complained of visual disturbances (retinitis). These conditions were grouped together as 'end organ diseases' with a poor outcome.

Key to advancing the treatment of HCMV in SOT was defining the natural history of HCMV infection. Infection with this virus was then seen to precede EOD and to rise to high viral loads before EOD occurred. This gave an opportunity for active infection to be treated before it caused EOD; termed pre-emptive therapy (PET).

A series of studies in the 1990s demonstrated that the highest viral loads in urine from patients with renal transplantation were found in the donor positive-recipient negative $(\mathrm{D}+\mathrm{R}-)$ combination; that is, the donor is infected with HCMV and the recipient has no natural immunity to this virus ${ }^{46}$. Importantly, this subgroup also had the highest proportion meeting a case definition ${ }^{41}$ of HCMV EOD (signs and symptoms together with HCMV detected histopathologically in a biopsy of the affected organ). Importantly, multivariate statistical models were consistent with a high viral load causing EOD, rather than the alternative interpretation of the absence of natural immunity being responsible ${ }^{46,47}$. Although elements of immune function are important, their contribution is captured by quantitative measures of viraemia ${ }^{47}$.

FIGURE 3a shows how the prevalence of IgG antibodies pre transplantation can group SOT into four subgroups. Post transplantation, primary infection occurs in $80 \%$ of cases of D+R- transplantation whereas in the D-R+ subgroup only $40 \%$ of recipients reactivate latent $\mathrm{HCMV}$ after transplantation ${ }^{9}$ (FIG. 3b). The most complicated group to study are the $\mathrm{D}+\mathrm{R}+$ subgroup (that is, both donor and recipient are infected with HCMV), who are at risk of either reactivation or reinfection and have an intermediate risk phenotype of $55 \%$ viraemia ${ }^{9}$. Under the simple assumption that their risk of reactivation is the same as those in the $\mathrm{D}-\mathrm{R}+$ subgroup, the rate of reinfection must approximate $15 \%$ (55\% in $\mathrm{D}+\mathrm{R}+$ minus $40 \%$ in $\mathrm{D}-\mathrm{R}+$ ). In cases when two recipients of renal transplantation ( $\mathrm{R}+$ and $\mathrm{R}-$ ) received kidneys from the same donors who are seropositive, sequencing formally proved reinfection where HCMV came from the donor ${ }^{48}$. Furthermore, the risk of EOD was found to be intermediate between that of the D+R-subgroup (high viral load and high risk of EOD) and the D-R+ subgroup (low viral load and low risk of EOD). In the future, sequencing strains of HCMV offers the potential of diagnosing reinfection and differentiating it from reactivation; studies are underway in the more straightforward subgroup to interpret, $\mathrm{D}+\mathrm{R}-{ }^{49}$.

The better outcomes in $\mathrm{R}+$ individuals overall clearly support a role for pre-existing immunity ${ }^{9}$. Comparison of FIG. $4 \mathrm{a}$ and FIG. $4 \mathrm{~b}$ shows that pre-existing natural immunity is moderately effective at preventing high peak viral loads, thereby explaining why EOD is much more common in the $\mathrm{D}+\mathrm{R}-$ subgroup than the $\mathrm{D}+\mathrm{R}+$ subgroup. However, natural immunity is not able to prevent low-level replication (FIG. 4C), which represents reactivation of latent infection.

End organ disease in stem cell transplantation. Evidence that viral load and natural immunity are an important component of EOD comes from studies of the other major transplantation cohort - individuals who have undergone SCT.

The first notable difference is that the high-risk group here is the D-R+ cohort (FIG. 3), and thus the opposite of individuals who have undergone SOT ${ }^{12}$. This view is supported by the sero-epidemiology, which suggests that transmission from a D+ organ is rare in this setting ${ }^{50}$. HCMV transmission is likely a function of the number of stem cells donated coupled with the very low frequency of latently infected cells $(<0.01 \%)$ predicted to be in the graft ${ }^{51}$. Additionally, in the $\mathrm{D}+$ setting the graft also transfers HCMV cell-mediated immunity to the recipient ${ }^{52}$. Thus, it follows that the adoptive transfer of HCMV immunity without a substantial increase in the risk of HCMV infection from a D+ individual leads to better outcomes than a D- graft into an $\mathrm{R}+$ individual. Experimentally, immunization of donors prior to bone marrow harvest can transfer immunity to recipients $s^{53}$.

Additionally, FIG. 3b shows that SCT patients have the same high risk of EOD as D+R-SOT patients despite the SCT patients being $\mathrm{R}+$. By contrast, the two $\mathrm{R}+\mathrm{SOT}$ subgroups have a low risk of EOD (FIG. 3b). This shows that $\mathrm{R}+$ SCT patients are more susceptible to EOD than $\mathrm{R}+\mathrm{SOT}$ patients, because there is a higher incidence and greater severity of EOD in this cohort. This is explained by SCT patients developing EOD at lower viral loads than SOT patients ${ }^{47,54,55}$ (FIG. 4d). 
The advent of pre-emptive therapy to manage HCMV infection in transplantation. What became clear from studies of both SOT and SCT cohorts is that viraemia is a robust biomarker to predict individuals most at risk of HCMV disease post transplantation ${ }^{56}$.

In both transplant groups, the incidence of EOD was found to have a non-linear relationship with viral load, such that disease was uncommon until a high viral load was reached, with SCT patients being more susceptible to EOD than SOT patients ${ }^{47,54}$. Importantly, all patients could be monitored and given antiviral drugs when a low threshold of viraemia was reached ${ }^{57}$. Such PET is highly effective at preventing EOD ${ }^{58}$ (FIG. 5). An alternative strategy of giving antiviral drugs prophylactically is also effective, but at the risk of delaying EOD until prophylaxis is stopped ${ }^{59,60}$ (FIG. 5). Both strategies

a

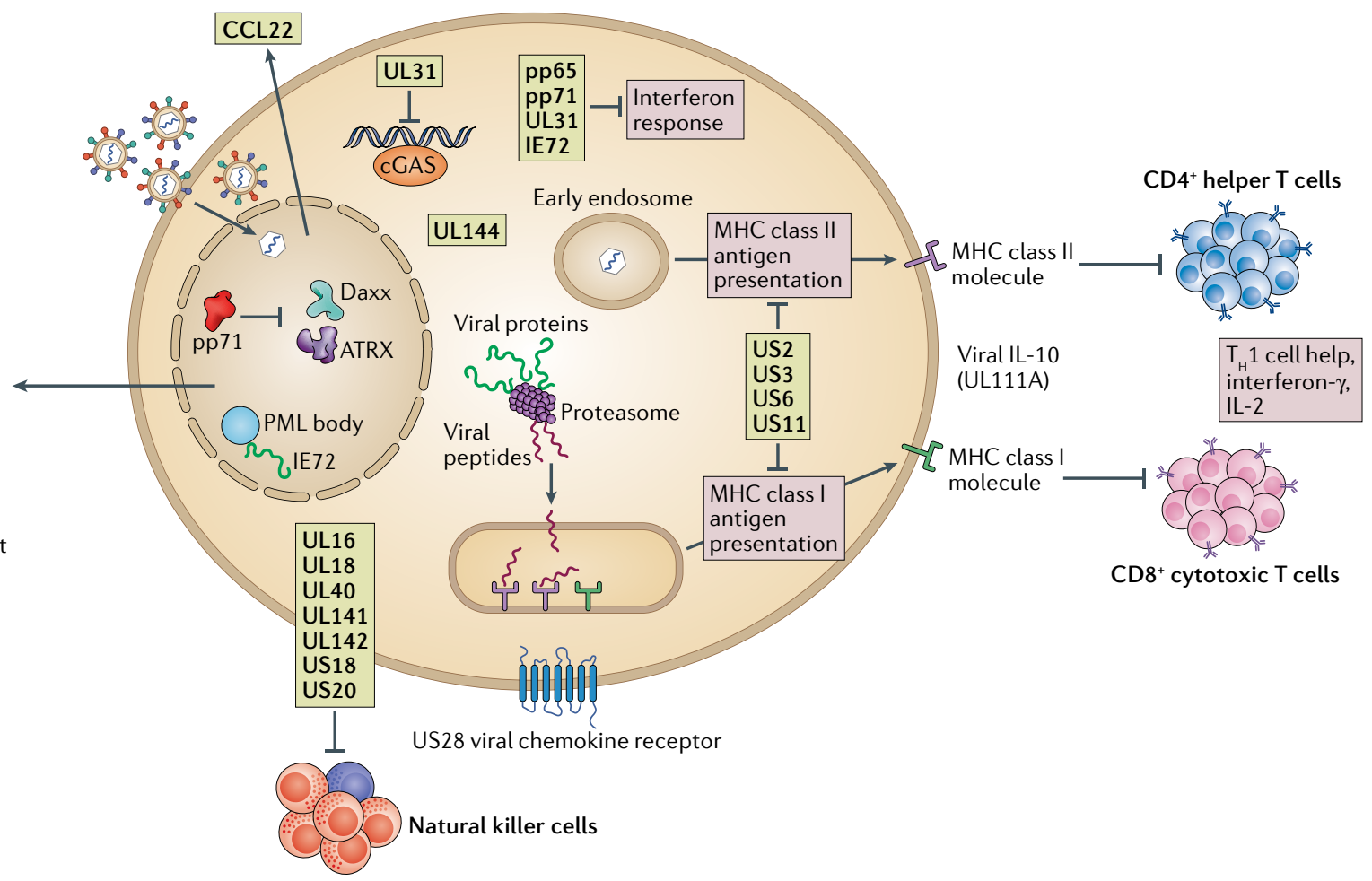

Viral $\alpha$-chemokines: UL128, UL146, UL147

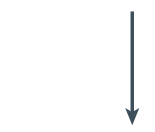

Neutrophil recruitment and activation

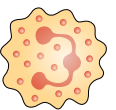

b

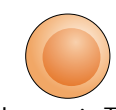

Allogeneic T cell

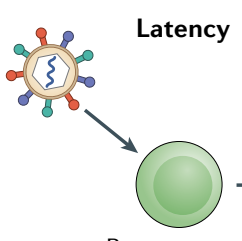

Bone marrow precursor

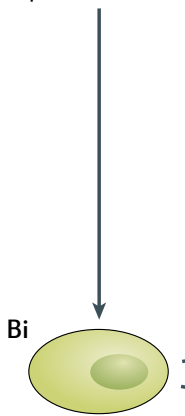

Endothelial progenitor dendritic cell progenitor
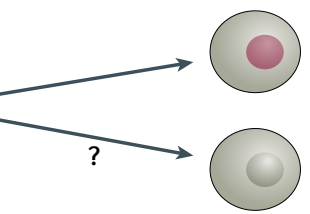

Venous endothelial cell
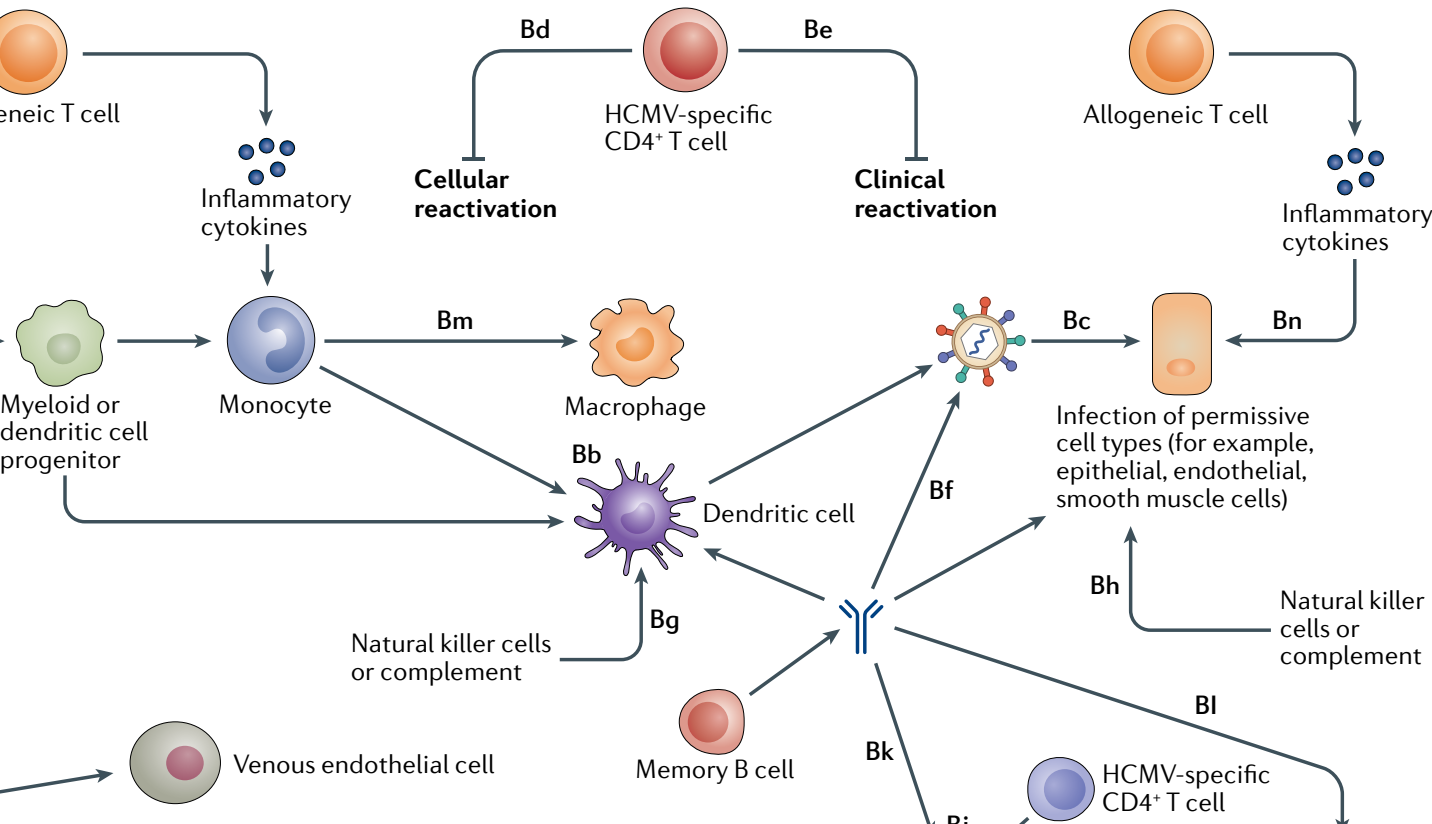

Aortic endothelial cell
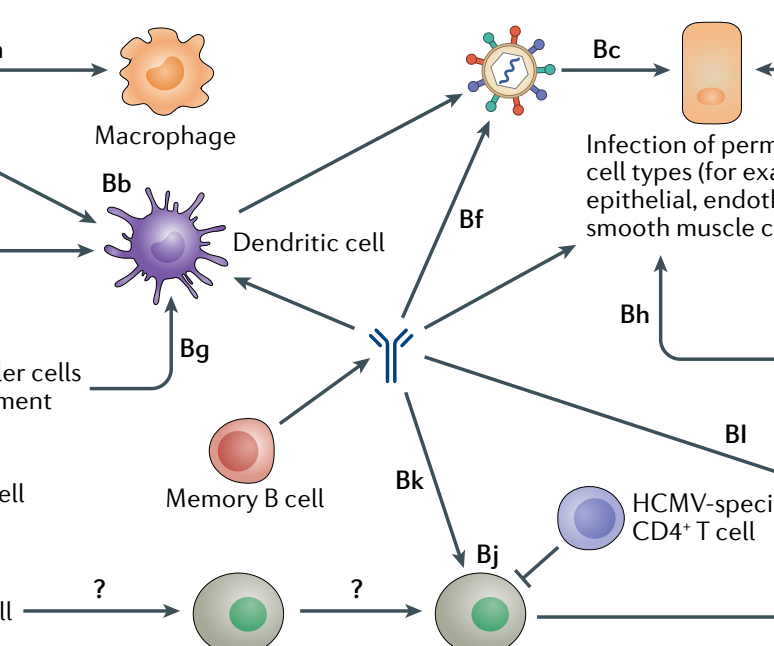

Infection of permissive cell types (for example, epithelial, endothelial, smooth muscle cells)

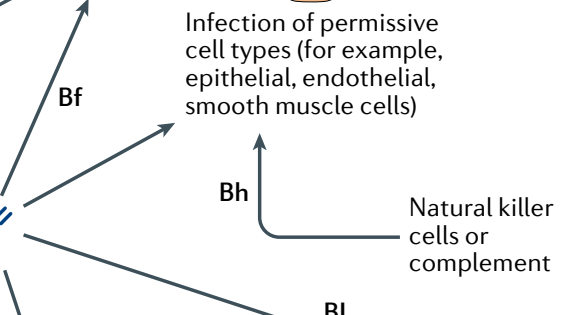
Tissue-resident
endothelial cell 
Leukoviraemia cytomegalovirus (HCMV) within the bloodstream that is attached to white blood cells or within them.
The presence of human

are recommended in current clinical guidelines for the management of SOT and have served to reduce the number of individuals experiencing $\mathrm{EOD}^{61,62}$. In a recent RCT, patients with liver transplantation randomized to management with PET had significantly less late-onset EOD than those managed with prophylaxis therapy ${ }^{62}$. The concept that PET allows low-level antigen presentation to the immune system is supported by studies of humoral and cell-mediated immunity post transplantation ${ }^{58}$. In other words, the low levels of viraemia that occur in individuals monitored by PET have a low risk of causing EOD yet are sufficient to stimulate the immune system to bring viraemia under control.

These quantitative studies characterized a series of parameters that can be used to define the severity of HCMV (proportion of individuals with viraemia, duration of viraemia and peak viral load). These parameters are now sufficiently robust to be accepted by regulators as end points for RCTs ${ }^{56}$. Importantly, these surrogate

4 Fig. 2 | Viral and host functions in human cytomegalovirus latency and reactivation a | In healthy individuals, a robust innate and adaptive immune response restricts human cytomegalovirus (HCMV) reactivation and replication. $\mathrm{HCMV}$ counters this with an armoury of measures to disable all arms of the immune response. Recognition by $\mathrm{CD} 8^{+}$ T cells is limited by major histocompatibility complex (MHC) class I downregulation and prevention of antigen loading and presentation at the cell surface. Similarly, MHC class II presentation to $\mathrm{CD} 4^{+} \mathrm{T}$ cells is prevented by similar strategies including the expression of a viral interleukin-10 (IL-10) homologue that promotes MHC class II downregulation. Loss of MHC class I can potentially activate natural killer cell recognition and killing according to the 'missing self hypothesis', thus HCMV promotes the expression of an HLA-E inhibitory receptor as well as numerous gene products that disable natural killer activating receptors and upregulate natural killer inhibitory receptors. The interferon response is disabled at multiple points of the viral life cycle. Specifically, HCMV gene products interfere with DNA sensing pathways to prevent activation including inhibitors of IFI16 (for example, pp65 and US28) and cGAS-STING (UL31 and pp71). Interferon signalling is also disabled via an interaction of IE72 with the signal transducer and activator of transcription (STAT) transcription factor. HCMV also modulates the bio-activity of cytokines through expression of $\beta$-chemokine receptors that bind and sequester host cytokines. Additionally, $\mathrm{HCMV}$ encodes numerous $\alpha$-chemokines that mimic CXCL1 and CXCL2 activity to modulate the recruitment to, and activity of, immune cells at the site of infection. $\mathbf{b}$ | Potential roles for immunosuppression in HCMV infection and reactivation. $\mathrm{HCMV}$ establishes latency in $\mathrm{CD} 34^{+}$progenitor cells. Myeloid or dendritic cell progenitor (step Ba) differentiation into macrophages or dendritic cells promotes cellular reactivation (step Bb), production of infectious virus, and subsequent infection and replication in multiple permissive tissue cells (step Bc). HCMV-specific T cells can recognize cellular reactivation (step Bd) or disseminated infection (step Be). Additionally, B cells produce virus-neutralizing antibodies (step Bf) or non-neutralizing antibodies that likely recognize viral cell surface antigens on reactivating cells (step Bb) or newly infected cells (step Bc). This will promote the recruitment of antibodydependent effector functions (steps $\mathrm{Bg}$ and $\mathrm{Bh}$ ) to target the infected cells. A second site of viral persistence is hypothesized to be tissue-resident endothelial cells (step Bi), although whether they are seeded via differentiation from a latently infected CD $34^{+}$ progenitor or by direct infection in tissue is unknown. Hypothetically, these latently infected endothelial cells are activated by an undefined stimulus and thus, following viral replication, can be recognized by immune responses mediated by T cells (step Bj) and B cells (steps Bk and Bl) as described for macrophages and dendritic cells. In the context of immunosuppression, pre-existing $T$ cell responses will be reduced; in individuals who are seropositive, this leads to reduced control of both cellular and clinical reactivation. In individuals who are seronegative experiencing primary infection (who have no latent HCMV reservoir in CD34 ${ }^{+}$cells), the major impact of immunosuppression is a reduction in the generation of new $T$ cell and $B$ cell responses, reducing control of replication in permissive cells (steps $\mathrm{Be}, \mathrm{Bf}$ and $\mathrm{Bh}$ ). These processes are likely exacerbated through inflammation (allogeneic T cells or co-infection), enhancing cellular reactivation and viral replication in individuals who are seropositive (steps $\mathrm{Bm}$ and $\mathrm{Bn}$ ) and viral replication in infected individuals who are seronegative (step Bn). $T_{H} 1$ cell, Thelper 1 cell. markers of EOD also allow the continued study of HCMV natural history and pathogenesis without compromising patient treatment. For example, they revealed that the replication dynamics of primary HCMV infection in vivo is very similar to that of HIV infection - and much quicker than anticipated based on studies of the development of cytopathic effects in in vitro culture models ${ }^{63,64}$.

All of these observations were assimilated into a dynamic model of HCMV infection and EOD. Within hours of transplantation, HCMV reactivates from the donor organ. This productive infection may be controlled by the local immune response (FIG. 2). If it is not, HCMV appears in the blood, allowing the virus to disseminate to multiple organs. Note that viraemia can be detected as a leukoviraemia or a plasma viraemia. Plasma viraemia (strictly plasma DNAaemia) consists of short, fragmented portions of HCMV naked DNA within blood $^{65}$.

Although it is clearly not infectious, plasma viraemia still acts as a good biomarker for taking decisions about PET $^{56,66}$. The physical state of the virus is not defined in the case of leukoviraemia. Separation techniques using magnetic beads followed by quantitative PCR for HCMV DNA revealed that polymorphonuclear leukocytes make the largest contribution to the overall viral load in blood, and that HCMV DNA and late mRNA transcripts can be found in monocytes, B cells and T cells, consistent with productive infection ${ }^{67}$. The response to ganciclovir was similar when HCMV DNA was measured in each of these cellular fractions of peripheral blood ${ }^{66}$. Viraemia is not a guarantee of EOD because immune responses (FIC. 2) at the level of each organ may be able to prevent blood-organ transmission of virus and/or the development of EOD. If these immune responses are insufficient, HCMV may rise to high levels, causing EOD through various potential pathological processes. For example, low levels of virus may not complete a full replicative cycle (FIG. 1) yet display HCMV antigens on cells to make them targets for immunopathological responses. There is some evidence for such responses in the lungs ${ }^{44}$, although a high viral load is also found in bronchoalveolar lavage fluid in individuals with extensive, established pneumonitis ${ }^{43}$. Higher levels of virus may lead to productive infection (FIG. 1) with lysis of target cells; the retina would be a potential site for this, although it may also be followed by immune recovery uveitis, which is an immunopathological condition ${ }^{42}$. In all cases, initial immunosuppression, which may involve steroids that are given to all SOT patients, has an effect by increasing the viral load in the blood ${ }^{68}$. By contrast, steroids given to treat graft rejections in SOT (or graft versus host disease in SCT) increase the risk of EOD by lowering the viral load required to cause disease (that is, steroids are statistically independent from a high viral load as a risk factor for EOD in multivariate models) $)^{47}$. In summary, there is evidence for both viral lysis and immunopathology contributing to EOD. However, invasive samples from the affected site are only available from the late stages of disease when early immunopathological responses may have been joined by lytic destruction of target cells producing a high viral load. 
Methylprednisolone

A potent steroid given in high doses intravenously to deplete lymphocytes capable of causing graft rejection.

Atherosclerosis A chronic inflammatory condition with proliferation of cells and accumulation of lipid that tends to reduce blood flow through the vessel.
End organ disease in individuals with HIV. HCMV retinitis presented as a major complication in the dawn of the AIDS epidemic, with EOD most likely to occur in individuals seropositive for HCMV (and thus a result of HCMV reactivation or reinfection ${ }^{69}$. Indeed, it is a startling clinical observation that retinitis accounts for $85 \%$ of EOD in individuals with HIV compared with only $1 \%$ of individuals in the transplant groups; with no proven explanation for this. A possible explanation is that damage to the blood-retina barrier due to HIV infection may facilitate HCMV gaining preferential access to that organ.

A major difference between these individuals and the transplant cohorts is the absence of a starting point equivalent to the date of transplant to indicate when the risk of HCMV EOD increases. The major indicator is when the $\mathrm{CD}^{+} \mathrm{T}$ cell count of individuals with HIV falls below 100 cells per microlitre of blood. In these

\section{Box 2 | Overview of the different immunocompromised groups}

The three groups of patients considered here are all immunocompromised, but for different reasons.

Candidates for solid organ transplantation (SOT) become immunocompromised because of the drugs given to suppress cell-mediated immunity that would otherwise cause graft rejection. The immunocompromised state is most profound immediately after transplant and frequently facilitates human cytomegalovirus (HCMV) viraemia, but moderates as these drugs are reduced in dose over weeks. Graft rejection episodes are treated with methylprednisolone, which increases the risk associated with any given viral load and maintains an immunocompromised state for weeks to months. Memory humoral immunity remains relatively intact, but responses to new antigens (seen for HCMV infection in donor positive-recipient negative ( $D+R-)$ SOT) are blunted by poor $\mathrm{T}$ cell help. Risk factors for a profound immunocompromised state include graft rejection and the immunosuppression required to control it.

Haematopoietic stem cell transplantation (SCT) patients become immunocompromised when their bone marrow is ablated by chemotherapy to make room for donor marrow to engraft. Individuals remain profoundly immunocompromised until this occurs, starting from 2-3 weeks after the procedure, but taking months to reach sufficient immunity to protect against HCMV. Evidence from cohort studies supports recovery of $C D 8^{+} T$ cells ${ }^{149-151}$ and $C D 4^{+} T$ cells ${ }^{151,152}$ contributing to reduced HCMV end organ disease (EOD). Data from cohort studies are also consistent with case series from several investigators using methodological improvements on the original case series $^{99}$ of adoptive transfer of T cells of various specificities ${ }^{153,154}$, but we are not aware of any double-blind, placebo-controlled randomized clinical trial (RCT) that has formally proven the safely and efficacy of adoptive transfer. The appearance of graft versus host disease, coupled with its treatment, further compromises the immune system. Humoral immunity to recall antigens (such as HCMV reactivating from $\mathrm{R}+$ patients) remains relatively intact because plasma cells are resistant to the chemotherapy. To illustrate how long it takes to establish a new immune system, patients are typically not given vaccines based on live attenuated viruses until 12 months post transplantation ( 24 months for those with graft versus host disease). Risk factors for profound immunocompromise include allogeneic rather than autologous transplant, unrelated donors rather than a sibling donor, a small number of bone marrow cells transferred (cord blood or T cell-depleted transplants) and a haploidentical rather than a fully human leukocyte antigen (HLA)-matched donor.

Individuals with HIV are immunocompromised because active HIV infection depletes $\mathrm{CD} 4{ }^{+} \mathrm{T}$ cells. Once the $\mathrm{CD} 4^{+} \mathrm{T}$ cell count in peripheral blood declines from 1,000 cells to 100 cells per microlitre of blood, individuals become at risk of developing HCMV EOD. There is no formal evidence of the specific immune functions whose decline allows active infection to occur, but they are assumed to be $\mathrm{CD} 4^{+}$and $\mathrm{CD} 8^{+} \mathrm{T}$ cells, by analogy with SCT patients discussed above. Maintaining the CD4 ${ }^{+} T$ cell count above 100 cells per microlitre of blood through lifelong antiretroviral therapy has virtually abolished HCMV EOD in individuals with HIV. Risk factors for profound immunocompromise include the non-availability of antiretroviral therapy (due to cost, healthcare access or patient choice). individuals, natural history studies showed (FIG. 4e) that HCMV becomes detectable and rises to high levels in the blood, similar to those found in D+R-SOT patients ${ }^{70}$. Thereafter, individuals are at risk of developing EOD, but the temporal dynamics are altered; instead of preceding EOD by weeks (SOT) or days (SCT), individuals with HIV can have high HCMV viral loads for months before developing $\mathrm{EOD}^{71}$. One possible explanation we can offer is that blood-organ barriers (apart from the retina) are better preserved in individuals with HIV than in the transplant groups.

The 'indirect effects' of HCMV infection. EOD associated with HCMV has been well described in numerous important patient populations. What is less clear are the associated 'indirect effects' of HCMV infection and replication. This term was coined by Rubin to describe the unexpected high prevalence of conditions such as accelerated atherosclerosis seen in cohort studies of patients with heart transplantation with active HCMV infection ${ }^{72}$. This condition was not unique to HCMV infection, but the virus increased its incidence. Potential mechanisms that could lead HCMV to contribute to atherosclerosis include systemic inflammation, monocyte activation, $\mathrm{T}$ cell stimulation and effects on the endothelium ${ }^{73}$.

The evidence for HCMV causing such phenomena comes from observations made in subjects enrolled in double-blind, placebo-controlled RCTs. For example, accelerated atherosclerosis was significantly reduced by prophylaxis with ganciclovir in patients with $\mathrm{D}+\mathrm{R}-$ heart transplantation ${ }^{74}$. Likewise, the incidence of biopsy-confirmed acute graft rejections after renal transplantation was significantly reduced in an RCT of high-dose valaciclovir in the D+R-subgroup, but not in the recipients who are seropositive ${ }^{75}$. It is often said that the low levels of viraemia found in patients managed by PET must increase their risk of graft rejection. In fact, a meta-analysis by the Cochrane collaboration of the RCTs conducted to compare PET with prophylaxis show no differences for graft rejection, graft survival or patient survival $^{76}$.

In the SCT population, high mortality linked to HCMV serostatus is observed, even in the absence of overt HCMV-driven EOD ${ }^{12}$. Death in SCT patients is divided into relapse-related (that is, recurrence of leukaemia) or transplant-related (for example, opportunistic infections). Recipients of transplants who are seropositive for HCMV have an increased transplant-related mortality that was reduced by acyclovir prophylaxis ${ }^{77,78}$. The interpretation of these observations was limited by the broad-acting nature of acyclovir, but subsequent studies with HCMV-specific letermovir significantly reduced mortality as a predefined secondary end point of the $\mathrm{RCT}^{79}$. Importantly, it was the ability of letermovir to prevent viraemia that conferred the statistical benefit of reduced mortality ${ }^{80}$.

As with SCT, the major indirect effect of HCMV infection in individuals with HIV is death not explained by $\mathrm{EOD}^{12,80}$. Interestingly, it is HCMV viraemia and $\mathrm{CD}^{+}{ }^{+} \mathrm{T}$ cell count, and not HIV loads, that are the correlates of mortality ${ }^{13}$. Consistent with this, systemic 
Pseudo-type formation The formation of a virus particle that contains structural elements from more than one virus; a typical example 'types' as the virus that shares the same surface proteins. exposure to ganciclovir in individuals experiencing their first episode of retinitis reduced mortality rates ${ }^{81}$. A meta-analysis of RCTs of acyclovir also showed a significant reduction in mortality ${ }^{82}$.

\section{a Prevalence of $\operatorname{lgG}$ antibodies}
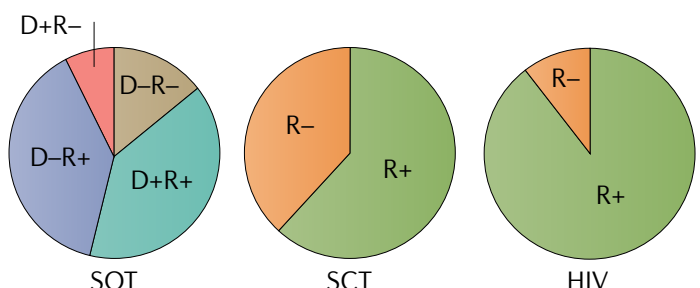

b Incidence of infection and disease

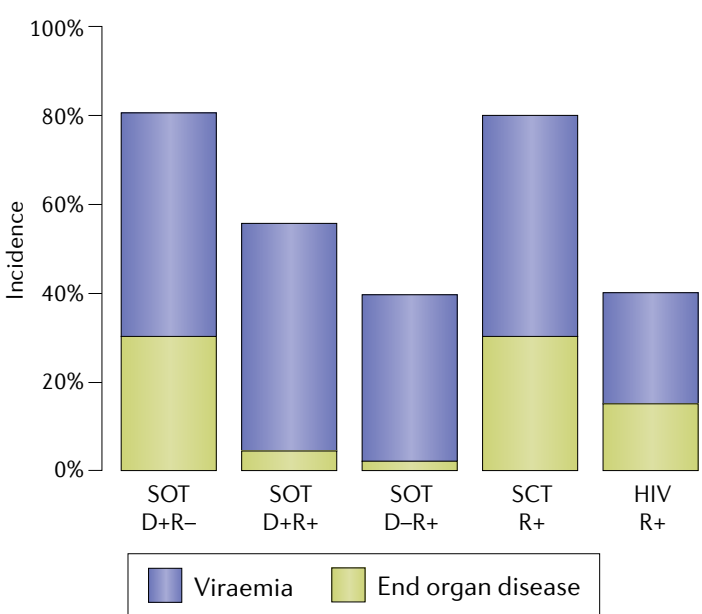

Fig. 3 | Prevalence of human cytomegalovirus antibodies and incidence of infection in immunocompromised individuals. a | Prevalence of prior human cytomegalovirus (HCMV) infection is high ( 90\%) in individuals with HIV infection and intermediate ( $60 \%)$ in those awaiting haematopoietic stem cell transplantation (SCT). The solid organ transplantation (SOT) patient group can be divided further according to prevalence of antibodies in the donor as well as the recipient. $\mathbf{b}$ |Once individuals become immunocompromised either through immunosuppression after transplantation or because the $\mathrm{CD} 4^{+} \mathrm{T}$ cell count in individuals who are HIV-positive falls below 100 cells per microlitre of blood, they are at risk of HCMV viraemia, which can be detected by PCR. In the SOT group, the risk is highest in those with primary infection (donor positiverecipient negative $(D+R-)$ ), intermediate in those at risk of reactivation or reinfection $(D+R+)$ and lowest in those at risk of reactivation only ( $D-R+)$. This illustrates that preexisting natural immunity against $\mathrm{HCMV}$ provides substantial protection against exogenous (reinfection) and endogenous (reactivation) sources of virus. Note that the incidence of end organ disease (EOD) declines in parallel with reduced detection of viraemia. In the SCT group, the risk of viraemia and EOD is as high as in $\mathrm{D}+\mathrm{R}$-individuals in the SOT group, despite the SCT patients being R+. Comparison of the SCT group with D-R+ individuals in the SOT group shows that reactivation dominates R+ SCT recipients and that ablation of their bone marrow greatly reduces immunity acquired in the past. The incidence of both viraemia and EOD is intermediate in individuals who are HIV-positive. Figure drawn from information provided in $\mathrm{REFS}^{9,13,50,70,120-122}$.
Mechanisms by which HCMV has been shown to interact with HIV in vitro are transactivation of HIV gene expression and pseudo-type formation, both of which require the two viruses to infect a single cell ${ }^{83}$. Four other mechanisms that require the two viruses to infect neighbouring cells are stimulation of cytokine release, antigen presentation, upregulation of CD4 or its co-receptor and induction of an alternative entry receptor for $\mathrm{HIV}^{83}$. The plausibility of these interactions occurring in vivo was supported by detecting the nucleic acid of both viruses in more than $50 \%$ of tissues sampled at autopsy ${ }^{84}$. However, no evidence was found to support the prediction that HIV loads increase in individuals who are HIV-positive co-infected with HCMV (reviewed in REF. ${ }^{83}$ ). Attention therefore moved to an alternative mechanism based on the induction by HCMV of an excess of immunocommitted $\mathrm{CD}^{+} \mathrm{T}$ cells as part of its contribution to the 'immune risk phenotype' or 'immunosenescence' that is associated with increased prevalence of atherosclerosis in individuals with HIV $^{17,85}$. The possibility of a causal relationship was supported by the observation that the level of HCMV-specific $\mathrm{CD}^{+} \mathrm{T}$ cells was decreased significantly in a small RCT of valganciclovir ${ }^{85}$.

Overall, these observations are strikingly similar to those in the SOT and SCT populations, but have been largely overlooked by the HIV research community despite similar results being reported every few years ${ }^{86}$. We continue to suggest that studies of both HIV and transplantation could potentially benefit from collaboration to explore and compare potential mechanisms; for example, the observed increase in the prevalence of atherosclerosis after SOT could be explained by the excess of inflammatory $\mathrm{T}$ cells that has been reduced in an RCT involving individuals with HIV. This is important because the total amount of morbidity caused by the indirect effects of HCMV may exceed that currently attributed to $\mathrm{EOD}^{10}$. Furthermore, clinicians should be aware that 'silent' HCMV infection may be predisposing various patients to adverse outcomes, including excess mortality in the general population, increased duration of ventilation when patients are admitted for intensive care following heart attacks, burns or sepsis and increased severity of COVID-19 (REFS ${ }^{87-91}$ ). The important principle is that underlying HCMV infection induces a long-term inflammatory bias that can contribute to other medical conditions without manifestation of its presence.

\section{Immune correlates of HCMV control}

What is clear from our understanding of clinical HCMV infection is that pathogenesis is mainly observed in individuals with poor immune responses. That said, the precise component of the immune response responsible for protection is still unclear. For example, active HCMV infection is seen in individuals with poor cell-mediated immunity measured against the MIE antigen or pp65 proteins - two immunodominant antigens ${ }^{92}$. However, it has been difficult to define cut-off levels at baseline or at the end of prophylaxis to identify which SOT patients are not at risk of infection ${ }^{92}$. This is partly because of the fluctuating risk seen with time, as some patients require additional immunosuppression in the form of 
steroids $^{47,68}$. It is also partly because clinicians wish to be informed preferentially about the highest-risk patients, yet these are the $\mathrm{D}+\mathrm{R}$ - subgroup where measurements of specific immunity in the recipient are undetectable ${ }^{92}$. Furthermore, studies of recipients who are seropositive often fail to differentiate between control of reactivation or reinfection. Recently, a single paper has produced substantial evidence focused on the $\mathrm{D}+\mathrm{R}+$ subgroup that immune responses to the MIE antigen detectable pre transplantation predict the risk of HCMV viraemia post transplantation ${ }^{93}$.
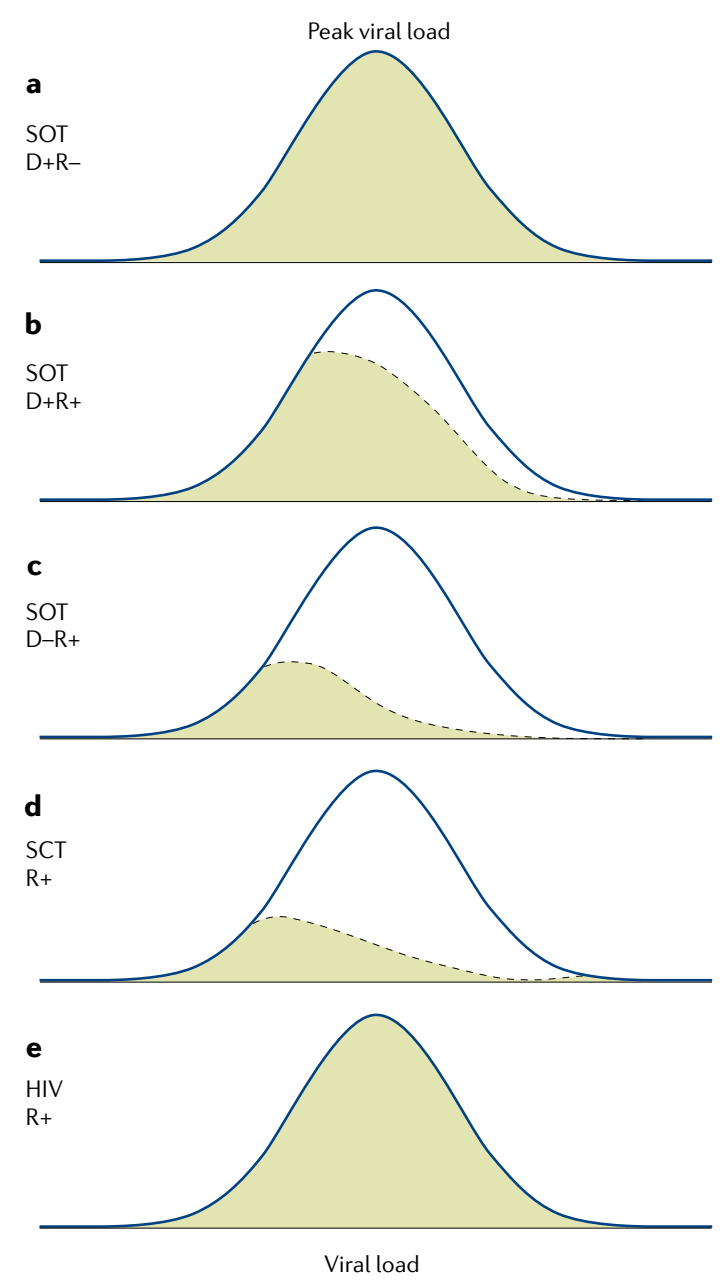

Fig. 4 | Distribution of peak viral loads in immunocompromised individuals. a | Peak viral loads approximate to a normal distribution in the donor positive-recipient negative ( $D+R-$ ) solid organ transplantation (SOT) patient subgroup. $\mathbf{b}$ | By contrast, the distribution is shifted strongly to the left in the $D+R+$ subgroup where the recipient has natural immunity pre transplantation (green). $\mathbf{c}$ | Natural immunity does not prevent low viral loads resulting from reactivation (or either reactivation or reinfection (part b)). d | Following haematopoietic stem cell transplantation (SCT), the peak viral loads are relatively low yet patients in this subgroup have a high risk of end organ disease (EOD) (see FIG. 3). This shows that SCT patients are susceptible to a low viral load that would be unlikely to cause EOD in SOT. e I Individuals with HIV have a high viral load distribution, similar to that seen after D+R-SOT. Figure drawn using data provided in $\mathrm{REFS}^{9,50,70,120-123}$.
One potential issue with current strategies is that focus has often centred on measuring the quantity rather than the quality of the immune response. This may not simply be a numbers game - it may be more a question of having the right response rather than a large response. A pivotal study demonstrated that the $\mathrm{T}$ cell response against $\mathrm{HCMV}$ is diverse and targeted against the full range of HCMV proteins ${ }^{94}$. These observations have been substantiated in numerous smaller, more focused studies that essentially demonstrate that the response is dynamic and broad ${ }^{17,95-98}$. Ongoing studies are addressing whether infusion of HCMV-specific T cells into patients can provide protection ${ }^{99}$.

Antibodies, natural killer cells and macrophages may theoretically contribute to a protective immune response and are expected to interact and cooperate with T cells to control HCMV replication. A very recent paper has reported some of the immune functions that require collaborative contributions from more than one component of the immune system by studying viral proteins expressed at the surface of the infected cell and determining which could mediate antibody-dependent cellular cytotoxicity ${ }^{100}$. Remarkably, these targets were not the major structural glycoproteins of the virus but the proteins it deploys as immune evasins $\mathrm{s}^{100}$. Future studies of this kind have the potential to give a more sophisticated assessment of the immune capability of individual patients at risk of HCMV infection.

\section{Emerging strategies to treat infection}

Ganciclovir was licensed in 1989 and remains the only licensed drug potent enough to treat active HCMV infection. Although the oral prodrug valganciclovir was licensed in 2001, it delivers the same active ingredient. For strains of HCMV resistant to ganciclovir, foscarnet is used off-label. Clearly, this field would benefit from more licensed drugs that are both safe and effective against HCMV. One important outcome of comprehensive studies of the natural history and pathogenesis of HCMV is the provision of strong evidence that measuring the viral load is a robust surrogate for measuring EOD, and thus a requirement for $\mathrm{PET}^{56}$. This becomes particularly important for clinical trials seeking to test the anti-HCMV activity of novel compounds.

Three phase II RCTs have been conducted in the context of SCT using PET as the read-out to determine whether novel antiviral drugs given prophylactically can control HCMV viraemia better than placebo ${ }^{101-103}$. The first drug was maribavir, which reduces the ability of the virus to break down the nuclear membrane required to allow newly formed virions to escape from the nucleus of an infected cell. The second drug was brincidofovir, which, similar to ganciclovir, inhibits the virus-encoded DNA polymerase. The third drug was letermovir, which inhibits the terminase complex that takes newly synthesized HCMV DNA in concatameric form, cuts it into genome lengths and packages these into nascent virions. All three drugs were successful without causing bone marrow suppression and proceeded to phase III studies.

In the first phase III study (maribavir), EOD was required as the primary end point. The drug failed to reduce EOD for two reasons: PET was allowed for 

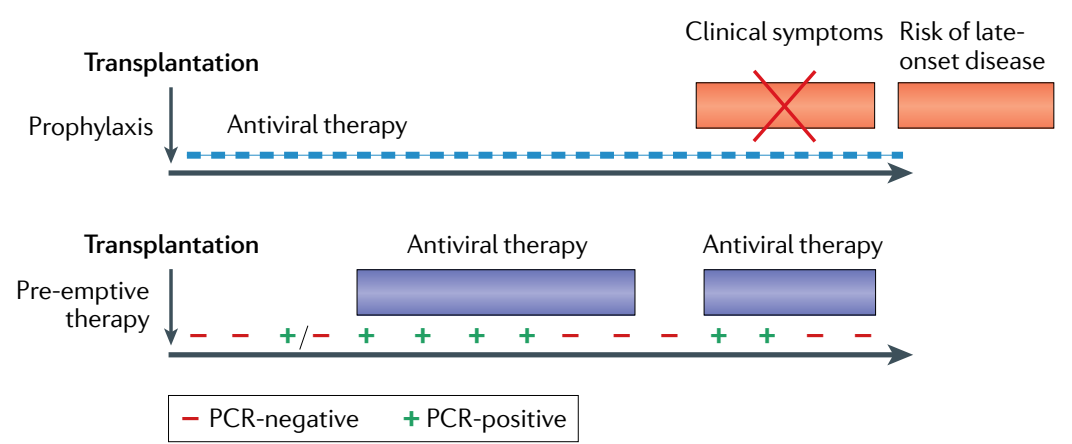

Fig. 5 | Two distinct strategies used to reduce human cytomegalovirus disease in allograft recipients. In the case of prophylaxis (upper panel), an antiviral drug is given from the time of transplantation (as soon as the patient can tolerate oral medication) for a fixed period of time, with clinical trials for solid organ transplantation (SOT) supporting durations of either 100 or 200 days $^{59,124}$. This strategy is effective in preventing end organ disease (EOD), but patients are at risk once again after prophylaxis is stopped, including with strains of human cytomegalovirus (HCMV) resistant to the drug used for prophylaxis (late-onset disease) ${ }^{125}$. In the case of pre-emptive therapy (PET) (lower panel), patients are monitored frequently to determine whether HCMV DNA is detectable by PCR. Individuals with low viral loads continue to be monitored, but those with a viral load above a defined threshold are given antiviral therapy until two consecutive blood tests can no longer detect HCMV DNA. Patients continue to be monitored and may require a subsequent episode of PET. Humoral and cell-mediated responses are superior in SOT managed using PET and late-onset disease is uncommon ${ }^{58,62}$. For haematopoietic stem cell transplantation (SCT), valganciclovir prophylaxis cannot be used because of bone marrow toxicity of the drug. Letermovir is safe enough to be used for prophylaxis ${ }^{79}$ and is combined with PET. If the individual fails to respond to treatment (that is, the viral load does not show at least a one-log reduction over 2 weeks), refractory HCMV infection is diagnosed. This may be due to poor host responses and/or the selection of strains resistant to the antiviral drug being administered. At present, foscarnet is commonly used to treat ganciclovir-resistant strains of HCMV, but has severe side effects.

Phase II results for maribavir are encouraging ${ }^{126}$ with phase III randomized clinical trial $(\mathrm{RCT})$ results expected in 2021.

patients in both arms of the study and rescued those who had failed prophylaxis; and the sponsors chose the lowest dose of drug instead of the highest non-toxic dose $\mathrm{e}^{104}$. In the second study (brincidofovir), PET was allowed as the primary end point, but a drug-free washout period was included after the end of prophylaxis. The drug initially suppressed the need for PET but this difference then declined with time, leaving no overall significant difference when compared with placebo ${ }^{105}$. The reason for this was an excess of graft versus host disease in the drug arm of the study that was treated with steroids that then precipitated HCMV viraemia. Many of these clinically diagnosed cases were not true graft versus host disease (which is classically diagnosed with diarrhoea, rash and abnormal liver function) but simply cases of diarrhoea caused as a known side effect of brincidofovir ${ }^{105}$. The third drug (letermovir) reduced PET significantly and was licensed for use ${ }^{79}$.

While these RCTs were in progress, regulators in the USA and the EU progressively accepted that EOD was an undesirable and impractical end point and that the need for PET was now appropriate for phase III studies ${ }^{56}$. Studies of future drugs should therefore now be more straightforward to conduct. Two other aspects of regulatory requirements for phase III studies now also need to be brought up to date. First, drugs for prophylaxis should be given immediately post transplantation rather than waiting for engraftment, which is a hangover from studying the bone marrow toxic drug ganciclovir. Second, there is no scientific rationale for requiring a washout period after prophylaxis ends before assessing whether the need for PET has been reduced. This is not a requirement for anti-HIV drugs and is another hangover from the original ganciclovir study. Thus, application of modern understanding of the natural history and pathogenesis of HCMV is rapidly improving clinical trial design.

\section{Evaluation of novel vaccines}

There is no doubt that the development of vaccines to protect against HCMV infection or disease will be complex. This virus can establish lifelong latency and immune individuals can experience repeat infections from endogenous (reactivation) or exogenous (reinfection) sources despite the host committing substantial immune resources against HCMV. An early RCT gave live attenuated Towne vaccine strain (BOX 1) or placebo to candidates who are seronegative awaiting renal transplantation ${ }^{106}$. Post transplantation, the incidence of HCMV infection and EOD was not reduced but the severity of EOD was. The subsequent development of quantitative PCR allowed the viral load parameters described above to be used as pharmacodynamic read-outs to determine whether vaccines have activity against HCMV replication in these patient populations.

A vaccine consisting of viral glycoprotein $B(\mathrm{gB})$ with MF59 adjuvant given prior to SOT showed reduced post-transplantation viral load parameters when compared with recipients of placebo ${ }^{107}$. The correlate of immune protection was the titre of IgG antibodies made against $\mathrm{gB}^{107}$. Subsequent detailed studies of responses against individual antigenic domains of $\mathrm{gB}$ proposed that antibodies against antigenic domain 2 helped protect recipients who are seropositive from viraemia $^{108,109}$. Antibodies against the immunodominant antigenic domain 1 were not protective, consistent with the possibility that the presence of this domain represents another example of HCMV evading protective immune responses. These hypotheses should be tested formally in future RCTs.

To test that antibodies were a mechanistic correlate of protection, Genentech evaluated placebo-controlled infusion of preformed monoclonal antibodies specific for HCMV at the time of D+R- renal transplantation ${ }^{110}$. The company conducted an RCT in 120 individuals and demonstrated significant interruption of transmission of HCMV from donor to recipient ${ }^{111}$. This approach of using active and passive immunization serially and in tandem in SOT should be applied to the evaluation of novel vaccines in the future ${ }^{112}$.

Disappointing results were recently presented orally with a DNA plasmid vaccine in SCT that appeared to be poorly immunogenic and did not reduce the need for PET. When these phase III study results are published, it will be important to determine whether the change from immunizing donors in the encouraging phase II study was important ${ }^{113}$.

Two more HCMV vaccines have proceeded to phase II studies. Hookipa Pharma presented a modified lymphocytic choriomeningitis virus construct to 
express either $\mathrm{gB}$ or pp65. Co-administration of both constructs produced good humoral and cell-mediated responses and the results of a phase II study in patients who are seronegative with renal transplantation are awaited ${ }^{114}$. Positive results from this study could lead to RCTs in women of childbearing age at risk of primary infection. Merck have engineered two proteins within HCMV strain Ad169 modified to express the pentameric complex by fusing two viral proteins (IE1/2 and pUL51) to the destabilizing domain of FK506-binding protein 12 . This fusion targets these essential proteins for degradation by the proteasome unless an exogenous chemical is present ${ }^{115}$. The resulting genetically inactivated whole virus strain is being studied in women of childbearing age who are seronegative but could easily be applied to immunocompromised individuals in the future. When the results of these two studies are published, it will be possible to review the evidence for reduced primary infection, examine the immune correlates of protection and make recommendations for whether either or both products should proceed to phase III studies. These studies will be larger versions of the current phase II studies, with at least 30,000 women who are seronegative required. The primary end point will also change from primary infection in the women to congenital infection in their neonates. We recommend that such studies in women and the SOT population should proceed in parallel because of the similarities of HCMV in both patient populations ${ }^{116}$. Meanwhile, the same and/or different vaccines should be studied for their ability to 'boost' or 'improve' the natural immune response to HCMV so that the incidence of reactivations or reinfections can be reduced. The SOT population routinely monitored by PCR and managed by PET represents an ideal population to study. We also recommend that studies of active immunization should proceed concurrently with studies of passive immunotherapy using monoclonal antibodies with defined reactivity against specific proteins of HCMV; the SOT population acts effectively as a human challenge model to facilitate such studies.

\section{Conclusions and open questions}

We have reviewed how a virus that does not declare its presence by producing specific symptoms can nevertheless be monitored prospectively to define quantitative parameters of replication. These measures can be deployed for PET to reduce EOD and to define immune correlates of control. By giving a prototype vaccine or placebo pre transplantation, the viral load parameters can be used as pharmacodynamic read-outs of successful protection. Passive transfer of monoclonal antibodies or $\mathrm{T}$ cells can then be used to both confirm the immune correlate and establish a medically acceptable new treatment. Clinical cohorts continue to report reduced survival of patients with grafts and/or allografts in subgroups at risk of active HCMV infection ${ }^{12,117}$, so the goal should be to return these parameters to the values found in the D-R- subgroup. Although HCMV represents a complex target, we are optimistic that serial rounds of iterative studies will finally bring this important and under-recognized human pathogen under control.

Published online 24 June 2021
1. Zuhair, M. et al. Estimation of the worldwide seroprevalence of cytomegalovirus: a systematic review and meta-analysis. Rev. Med. Virol. 29, e2034 (2019).

2. Pembrey, L. et al. Seroprevalence of cytomegalovirus, Epstein Barr virus and varicella zoster virus among pregnant women in Bradford: a cohort study. PLOS ONE 8, e81881 (2013).

3. Pembrey, L. et al. Cytomegalovirus, Epstein-Barr virus and varicella zoster virus infection in the firs two years of life: a cohort study in Bradford, UK. BMC Infect. Dis. 17, 220 (2017).

4. Staras, S. A. et al. Cytomegalovirus seroprevalence and childhood sources of infection: a populationbased study among pre-adolescents in the United States. J. Clin. Virol. 43, 266-271 (2008).

5. Griffiths, P. D., McLean, A. \& Emery, V. C. Encouraging prospects for immunisation against primary cytomegalovirus infection. Vaccine 19, 1356-1362 (2001).

This article presents the first calculation of the basic reproductive number for HCMV, showing that only $60 \%$ of the population have to be rendered immune to achieve herd immunity.

6. Colugnati, F. A., Staras, S. A., Dollard, S. C. \& Cannon, M. J. Incidence of cytomegalovirus infection among the general population and pregnant women in the United States. BMC Infect. Dis. 7, 71 (2007).

7. Mayer, B. T. et al. Transient Oral Human Cytomegalovirus Infections Indicate Inefficient Viral Spread from Very Few Initially Infected Cells. J. Virol. 91, e00380-17 (2017).

8. Staras, S. A. et al. Influence of sexual activity on cytomegalovirus seroprevalence in the United States, 1988-1994. Sex. Transm. Dis. 35, 472-479 (2008).

Atabani, S. F. et al. Cytomegalovirus replication kinetics in solid organ transplant recipients managed by preemptive therapy. Am. J. Transplant. 12, 2457-2464 (2012)

This article presents a large cohort study of patients with renal or liver transplantation managed exclusively with PET showing multiple parameters of HCMV viral load.
10. Griffiths, P. The direct and indirect consequences of cytomegalovirus infection and potential benefits of vaccination. Antivir Res. 176, 104732 (2020).

11. Cannon, M. J. \& Davis, K. F. Washing our hands of the congenital cytomegalovirus disease epidemic BMC Public Health 5, 70 (2005).

This article clearly demonstrates that HCMV is less well recognized by the general public than other conditions that are medically less important.

12. Boeckh, M. \& Nichols, W. G. The impact of cytomegalovirus serostatus of donor and recipient before hematopoietic stem cell transplantation in the era of antiviral prophylaxis and preemptive therapy. Blood 103, 2003-2008 (2004).

This article clearly describes the excess mortality seen in SCT patients with HCMV.

13. Deayton, J. R. et al. Importance of cytomegalovirus viraemia in risk of disease progression and death in HIV-infected patients receiving highly active antiretroviral therapy. Lancet 363, 2116-2121 (2004). This prospective cohort study shows that mortality in individuals who are HIV-positive is driven by the CD4+ T cell count and HCMV rather than by HIV

14. Fielding, C. A. et al. Control of immune ligands by members of a cytomegalovirus gene expansion suppresses natural killer cell activation. Elife 6 e22206 (2017).

15. Patel, M et al. HCMV-encoded NK modulators: lessons from in vitro and in vivo genetic variation. Front. Immunol. 9, 2214 (2018).

16. Berry, R., Watson, G. M., Jonjic, S., Degli-Esposti, M. A $\&$ Rossjohn, J. Modulation of innate and adaptive immunity by cytomegaloviruses. Nat. Rev. Immunol. 20, 113-127 (2020)

17. Jackson, S. E. et al. Latent cytomegalovirus (CMV) infection does not detrimentally alter T cell responses in the healthy old, but increased latent CMV carriage is related to expanded CMV-specific T cells. Front. Immunol. 8, 733 (2017)

18. Prod'homme, V. et al. The human cytomegalovirus MHC class I homolog UL 18 inhibits LIR-1 ${ }^{+}$but activates LIR-1- NK cells. J. Immunol. 178, 4473-4481 (2007).
19. Prod'homme, V. et al. Human cytomegalovirus UL141 promotes efficient downregulation of the natural killer cell activating ligand CD112. J. Gen. Virol. 91 2034-2039 (2010).

20. Kubin, M. et al. ULBP1, 2, 3: novel MHC class Irelated molecules that bind to human cytomegalovirus glycoprotein UL16, activate NK cells. Eur. J. Immunol. 31, 1428-1437 (2001).

21. Tomasec, P. et al. Downregulation of natural killer cellactivating ligand CD155 by human cytomegalovirus UL141. Nat. Immunol. 6, 181-UL188 (2005).

22. Cosman, D. et al. ULBPs, novel MHC class I-related molecules, bind to CMV glycoprotein UL16 and stimulate NK cytotoxicity through the NKG2D receptor. Immunity 14, 123-133 (2001).

23. Lilley, B. N., Ploegh, H. L. \& Tirabassi, R. S. Human cytomegalovirus open reading frame TRL11/IRL11 encodes an immunoglobulin G Fc-binding protein J. Virol. 75, 11218-11221 (2001).

24. Atalay, R. et al. Identification and expression of human cytomegalovirus transcription units coding for two distinct $\mathrm{Fc} \gamma$ receptor homologs. J. Virol. 76 8596-8608 (2002)

25. McSharry, B. P., Avdic, S. \& Slobedman, B. Human cytomegalovirus encoded homologs of cytokines, chemokines and their receptors: roles in immunomodulation. Viruses. 4, 2448-2470 (2012).

26. Klenerman, P. \& Hill, A. T cells and viral persistence: lessons from diverse infections. Nat. Immunol. 6 873-879 (2005).

27. Goodrum, F. Human cytomegalovirus latency: approaching the Gordian knot. Annu. Rev. Virol. 3, 333-357 (2016).

28. Dupont, L. \& Reeves, M. B. Cytomegalovirus latency and reactivation: recent insights into an age old problem. Rev. Med. Virol. 26, 75-89 (2016).

29. Jarvis, M. A. \& Nelson, J. A. Human cytomegalovirus persistence and latency in endothelial cells and macrophages. Curr. Opin. Microbiol. 5, 403-407 (2002).

30. Soderberg-Naucler, C., Fish, K. N. \& Nelson, J. A Reactivation of latent human cytomegalovirus by 
allogeneic stimulation of blood cells from healthy donors. Cell 91, 119-126 (1997).

31. Taylor-Wiedeman, J., Sissons, P. \& Sinclair, J. Induction of endogenous human cytomegalovirus gene expression after differentiation of monocytes from healthy carriers. J. Virol. 68, 1597-1604 (1994).

32. Reeves, M. B., MacAry, P. A., Lehner, P. J., Sissons, J. C. \& Sinclair, J. H. Latency, chromatin remodeling, and reactivation of human cytomegalovirus in the dendritic cells of healthy carriers. Proc. Natl Acad. Sci. USA 102, 4140-4145 (2005).

33. Hahn, G., Jores, R. \& Mocarski, E. S. Cytomegalovirus remains latent in a common precursor of dendritic and myeloid cells. Proc. Natl Acad. Sci. USA 95 3937-3942 (1998)

34. Lathey, J. L. \& Spector, S. A. Unrestricted replication of human cytomegalovirus in hydrocortisone-treated macrophages. J. Virol. 65, 6371-6375 (1991).

35. Weinshenker, B. G., Wilton, S. \& Rice, G. P. Phorbol ester-induced differentiation permits productive human cytomegalovirus infection in a monocytic cell line. J. Immunol. 140, 1625-1631 (1988).

36. Prosch, S., Docke, W. D., Reinke, P., Volk, H. D. \& Kruger, D. H. Human cytomegalovirus reactivation in bone-marrow-derived granulocyte/monocyte progenitor cells and mature monocytes. Intervirology 42, 308-313 (1999).

37. Reeves, M. B. $\&$ Compton, T. Inhibition of inflammatory interleukin- 6 activity via extracellular signal-regulated kinase-mitogen-activated protein kinase signaling antagonizes human cytomegalovirus reactivation from dendritic cells. J. Virol. 85, 12750-12758 (2011)

38. Hargett, D. \& Shenk, T. E. Experimental human cytomegalovirus latency in CD $14^{+}$monocytes. Proc. Nat Acad. Sci. USA 107, 20039-20044 (2010).

39. Mansfield, S., Griessl, M., Gutknecht, M. \& Cook, C. H. Sepsis and cytomegalovirus: foes or conspirators? Med. Microbiol. Immunol. 204, 431-437 (2015)

40. Li, X. et al. Cytomegalovirus infection and outcome in immunocompetent patients in the intensive care unit: a systematic review and meta-analysis. BMC Infect. Dis. 18, 289 (2018).

41. Ljungman, P. et al. Definitions of cytomegalovirus infection and disease in transplant patients for use in clinical trials. Clin. Infect. Dis. 64, 87-91 (2017).

42. Holland, G. N. Immune recovery uveitis. Ocul. Immunol. Inflamm. 7, 215-221 (1999).

43. Boeckh, $\mathrm{M}$. et al. Cytomegalovirus (CMV) DNA quantitation in bronchoalveolar lavage fluid from hematopoietic stem cell transplant recipients with CMV pneumonia. J. Infect. Dis. 215, 1514-1522 (2017).

This prospective study collects bronchoalveola lavage samples from SCT patients with HCMV pneumonitis and those who were well to demonstrate the high viral load associated with pneumonitis.

44. Grundy, J. E., Shanley, J. D. \& Griffiths, P. D Is cytomegalovirus interstitial pneumonitis in transplant recipients an immunopathological condition? Lancet 2, 996-999 (1987).

45. Hill, R. B. Jr., Rowlands, D. T. Jr. \& Rifkind, D. Infectious pulmonary disease in patients receiving immunosuppressive therapy for organ transplantation. N. Engl. J. Med. 271, 1021-1027 (1964)

46. Cope, A. V. et al. Quantity of cytomegalovirus viruria is a major risk factor for cytomegalovirus disease after renal transplantation. J.Med.Virol. 52, 200-205 (1997).

47. Cope, A. V. et al. Interrelationships among quantity of human cytomegalovirus (HCMV) DNA in blood, donor-recipient serostatus, and administration of methylprednisolone as risk factors for HCMV disease following liver transplantation. J. Infect. Dis. 176, 1484-1490 (1997).

48. Grundy, J. E. et al. Symptomatic cytomegalovirus infection in seropositive kidney recipients: reinfection with donor virus rather than reactivation of recipient virus. Lancet 2, 132-135 (1988)

This study of multiple recipients from groups of kidney donors shows how typing of HCMV strains can prove reinfection of recipients who are seropositive.

49. Hasing, M. E., Pang, X. L., Mabilangan, C. \& Preiksaitis, J. K. Donor cytomegalovirus transmission patterns in solid organ transplant recipients with primary infection. J. Infect. Dis. 223, 827-837 (2021)

50. Panagou, E. et al. Cytomegalovirus pre-emptive therapy after hematopoietic stem cell transplantation in the era of real-time quantitative PCR: comparison with recipients of solid organ transplants. Transpl. Infect. Dis. 18, 405-414 (2016).
51. Slobedman, B. \& Mocarski, E. S. Quantitative analysis of latent human cytomegalovirus. J. Virol. 73, 4806-4812 (1999)

52. Grob, J. P. et al. Immune donors can protect marrowtransplant recipients from severe cytomegalovirus infections. Lancet 1, 774-776 (1987).

53. Wimperis, J. Z. et al. Transfer of a functioning humoral immune system in transplantation of T-lymphocytedepleted bone marrow. Lancet 1, 339-343 (1986)

54. Gor, D. et al. Longitudinal fluctuations in cytomegalovirus load in bone marrow transplant patients: relationship between peak virus load, donor recipient serostatus, acute GVHD and CMV disease. Bone Marrow Transpl. 21, 597-605 (1998).

55. Duke, E. R. et al. Cytomegalovirus viral load kinetics as surrogate endpoints after allogeneic transplantation. J. Clin. Invest. 131, 1 (2020).

56. Natori, Y. et al. Use of viral load as a surrogate marker in clinical studies of cytomegalovirus in solid organ transplantation: a systematic review and meta-analysis. Clin. Infect. Dis. 66, 617-631 (2018).

This systematic analysis of the published literature shows that the HCMV viral load meets the predefined criteria laid down by regulators to be accepted as a surrogate biomarker for EOD.

57. Griffiths, P. D. et al. Randomized controlled trials to define viral load thresholds for cytomegalovirus pre-emptive therapy. PLOS ONE 11, e0163722 (2016).

58. Limaye, A. P. et al. Prospective assessment of cytomegalovirus immunity in high-risk donor seropositive/recipient-seronegative liver transplant recipients receiving either preemptive therapy or antiviral prophylaxis. J. Infect. Dis. 220, 752-760 (2019).

59. Humar, A. et al. The efficacy and safety of 200 days valganciclovir cytomegalovirus prophylaxis in high-risk kidney transplant recipients. Am. J Transpl. 10 1228-1237 (2010)

60. Limaye, A. P. et al. Late-onset cytomegalovirus disease in liver transplant recipients despite antiviral prophylaxis. Transplantation 78, 1390-1396 (2004).

61. Kotton, C. N. et al. The third international consensus guidelines on the management of cytomegalovirus in solid organ transplantation. Transplantation 102 900-931 (2018).

62. Singh, N. et al. Effect of preemptive therapy vs antiviral prophylaxis on cytomegalovirus disease in seronegative liver transplant recipients with seropositive donors: a randomized clinical trial. JAMA 323, 1378-1387 (2020)

This randomized study shows that PET significantly reduces late-onset disease compared with antiviral prophylaxis when used to manage patients with liver transplantation.

63. Emery, V. C., Cope, A. V., Bowen, E. F., Gor, D. \& Griffiths, P. D. The dynamics of human cytomegalovirus replication in vivo. J. Exp. Med. 190, 177-182 (1999).

64. Emery, V. C., Hassan-Walker, A. F., Burroughs, A. K \& Griffiths, P. D. Human cytomegalovirus (HCMV) replication dynamics in HCMV-naive and -experienced immunocompromised hosts. J. Infect. Dis. 185 1723-1728 (2002).

65. Tong, Y., Pang, X. L., Mabilangan, C. \& Preiksaitis, J. K. Determination of the biological form of human cytomegalovirus DNA in the plasma of solid-organ transplant recipients. J. Infect. Dis. 215, 1094-1101 (2017).

66. Razonable, R. R. et al. The clinical use of various blood compartments for cytomegalovirus (CMV) DNA quantitation in transplant recipients with CMV disease. Transplantation 73, 968-973 (2002)

67. Hassan-Walker, A. F., Mattes, F. M., Griffiths, P. D. \& Emery, V. C. Quantity of cytomegalovirus DNA in different leukocyte populations during active infection in vivo and the presence of $\mathrm{gB}$ and UL18 transcripts. J. Med. Virol. 64, 283-289 (2001)

68. Van Damme, E. et al. Glucocorticosteroids trigger reactivation of human cytomegalovirus from latently infected myeloid cells and increase the risk for HCMV infection in D+R+ liver transplant patients. J. Gen. Virol. 96, 131-143 (2015)

69. Centers for Disease Control. Kaposi's sarcoma and pneumocystis pneumonia among homosexual men - New York City and California. MMWR 30 305-308 (1981)

70. Bowen, E. F. et al. Cytomegalovirus (CMV) viraemia detected by polymerase chain reaction identifies a group of HIV-positive patients at high risk of CMV disease. AIDS 11, 889-893 (1997).
71. Bowen, E. F. et al. Natural history of untreated cytomegalovirus retinitis. Lancet 346, 1671-1673 (1995).

72. Rubin, R. H. The indirect effects of cytomegalovirus infection on the outcome of organ transplantation. JAMA 261, 3607-3609 (1989).

This editorial by an experienced infectious disease physician explains how HCMV is associated with indirect effects post transplantation.

73. Vasilieva, E., Gianella, S. \& Freeman, M. L. Novel strategies to combat CMV-related cardiovascular disease Pathog Immun 5, 240-274 (2020).

74. Valantine, H. A. et al. Impact of prophylactic immediate posttransplant ganciclovir on developmen of transplant atherosclerosis: a post hoc analysis of a randomized, placebo-controlled study. Circulation 100, 61-66 (1999).

75. Lowance, D. et al. Valacyclovir for the prevention of cytomegalovirus disease after renal transplantation International Valacyclovir Cytomegalovirus Prophylaxis Transplantation Study Group. N. Engl. J. Med. 340 , 1462-1470 (1999).

76. Owers, D. S., Webster, A. C. Strippoli, G. F. Kable, K. \& Hodson, E. M. Pre-emptive treatment for cytomegalovirus viraemia to prevent cytomegalovirus disease in solid organ transplant recipients. Cochrane Database. Syst. Rev. 2, CD005133 (2013).

77. Meyers, J. D. et al. Acyclovir for prevention of cytomegalovirus infection and disease after allogeneic marrow transplantation. N. Engl. J. Med. 318, 70-75 (1988).

78. Prentice, H. G. et al. Impact of long-term acyclovir on cytomegalovirus infection and survival after allogeneic bone marrow transplantation. European Acyclovir for CMV Prophylaxis Study Group. Lancet 343, 749-753 (1994).

79. Marty, F. M. et al. Letermovir prophylaxis for cytomegalovirus in hematopoietic-cell transplantation. N. Engl. J. Med. 377, 2433-2444 (2017).

80. Ljungman, P. et al. A mortality analysis of letermovir prophylaxis for cytomegalovirus (CMV) in CMVseropositive recipients of allogeneic hematopoietic-cell transplantation. Clin. Infect. Dis. 70, 1525-1533 (2019).

81. Kempen, J. H. et al. Mortality risk for patients with cytomegalovirus retinitis and acquired immune deficiency syndrome. Clin. Infect. Dis. 37, 1365-1373 (2003).

82. Ioannidis, J. P. et al. Clinical efficacy of high-dose acyclovir in patients with human immunodeficiency virus infection: a meta-analysis of randomized individual patient data. J. Infect. Dis. 178, 349-359 (1998).

83. Griffiths, P. D. Studies of viral co-factors for human immunodeficiency virus in vitro and in vivo. J. Gen. Virol. 79, 213-220 (1998).

84. Webster, A., McLaughlin, J. E., Johnson, M. A Emery, V. C. \& Griffiths, P. D. Use of the polymerase chain reaction to detect genomes of human immunodeficiency virus and cytomegalovirus in post-mortem tissues. J. Med. Virol. 47, 23-28 (1995).

85. Hunt, P. W. et al. Valganciclovir reduces T cell activation in HIV-infected individuals with incomplete CD4 ${ }^{+} \mathrm{T}$ cell recovery on antiretroviral therapy. J. Infect Dis. 203, 1474-1483 (2011).

86. Gianella, S. et al. Presence of asymptomatic cytomegalovirus and Epstein-Barr virus DNA in blood of persons with HIV starting antiretroviral therapy is associated with non-AIDS clinical events. AIDS 34 , 849-857 (2020).

87. Simanek, A. M. et al. Seropositivity to cytomegalovirus, inflammation, all-cause and cardiovascular diseaserelated mortality in the United States. PIOS ONE 6, e16103 (2011)

This article is the first large cohort study to examine and enumerate the excess mortality associated with HCMV in the general population.

88. Gkrania-Klotsas, E. et al. Seropositivity and higher immunoglobulin $\mathrm{G}$ antibody levels against cytomegalovirus are associated with mortality in the population-based European Prospective Investigation of Cancer - Norfolk cohort. Clin. Infect. Dis. 56 1421-1427 (2013).

89. Limaye, A. P. et al. Effect of ganciclovir on IL-6 levels among cytomegalovirus-seropositive adults with critical illness: a randomized clinical trial. JAMA 318 731-740 (2017)

90. Kadambari, S., Klenerman, P. \& Pollard, A. J. Why the elderly appear to be more severely affected by COVID-19: the potential role of immunosenescence and CMV. Rev. Med. Virol. 30, e2144 (2020). 
91. Shrock, E. et al. Viral epitope profiling of COVID-19 patients reveals cross-reactivity and correlates of severity. Science 71, 2375-2385 (2020).

92. Kumar, D. et al. A prospective multicenter observational study of cell-mediated immunity as a predictor for cytomegalovirus infection in kidney transplant recipients. Am. J. Transpl. 19, 2505-2516 (2019)

93. Jarque, M. et al. Cellular immunity to predict the risk of cytomegalovirus infection in kidney transplantation a prospective, interventional, multicenter clinical trial. Clin. Infect. Dis. 71, 2375-2385 (2020).

This article is the first study to show convincingly that measures of cell-mediated immunity at baseline could predict future episodes of viraemia in individuals post transplant.

94. Sylwester, A. W. et al. Broadly targeted human cytomegalovirus-specific $\mathrm{CD}^{+}$and $\mathrm{CD} 8^{+} \mathrm{T}$ cells dominate the memory compartments of exposed subjects. J. Exp. Med. 202, 673-685 (2005). This extensive, comprehensive study shows that the immune system responds to the entire set of proteins encoded by HCMV.

95. Jackson, S. E., Mason, G. M., Okecha, G., Sissons, J. G. \&Wills, M. R. Diverse specificities, phenotypes, and antiviral activities of cytomegalovirus-specific CD8 ${ }^{+}$T cells. J. Virol. 88, 10894-10908 (2014).

96. Jackson, S. E., Sedikides, G. X., Okecha, G. \& Wills, M. R. Generation, maintenance and tissue distribution of T cell responses to human cytomegalovirus in lytic and latent infection. Med. Microbiol. Immunol. 208, 375-389 (2019).

97. Elkington, R. et al. Ex vivo profiling of CD8 $8^{-}$-T-cell responses to human cytomegalovirus reveals broad and multispecific reactivities in healthy virus carriers J. Virol. 77, 5226-5240 (2003).

98. Sylwester, A. et al. A new perspective of the structural complexity of HCMV-specific T-cell responses. Mech. Ageing Dev. 158, 14-22 (2016).

99. Walter, E. A. et al. Reconstitution of cellular immunity against cytomegalovirus in recipients of allogeneic bone marrow by transfer of T-cell clones from the donor. N. Engl. J. Med. 333, 1038-1044 (1995). This article is the first demonstration that $T$ cells specific for HCMV could safely be adoptively transferred in SCT.

100. Vlahava, V. M. et al. Monoclonal antibodies targeting nonstructural viral antigens can activate ADCC against human cytomegalovirus. J. Clin. Invest. 131 https://doi.org/10.1172/JCI139296 (2021). This article is the first demonstration that antibody-dependent cellular toxicity is directed against the immune evasin proteins of HCMV rather than structural glycoproteins.

101. Winston, D. J. et al. Maribavir prophylaxis for prevention of cytomegalovirus infection in allogeneic stem-cell transplant recipients: a multicenter, randomized, double-blind, placebo-controlled, dose-ranging study. Blood 111, 5403-5410 (2008).

102. Marty, F. M. et al. CMX001 to prevent cytomegalovirus disease in hematopoietic-cell transplantation. N. Engl. J. Med. 369, 1227-1236 (2013).

103. Chemaly, R. F. et al. Letermovir for cytomegalovirus prophylaxis in hematopoietic-cell transplantation. N. Eng. J.Med. 370, 1781-1789 (2014).

104. Marty, F. M. et al. Maribavir prophylaxis for prevention of cytomegalovirus disease in recipients of allogeneic stem-cell transplants: a phase 3 double-blind, placebo-controlled, randomised trial. Lancet Infect.Dis. 11, 284-292 (2011).

105. Marty, F. M. et al. A randomized, double-blind, placebo-controlled phase 3 trial of oral brincidofovir for cytomegalovirus prophylaxis in allogeneic hematopoietic cell transplantation. Biol. Blood Marrow Transpl. 25, 369-381 (2019).

106. Plotkin, S. A. et al. Towne-vaccine-induced prevention of cytomegalovirus disease after renal transplants. Lancet 1, 528-530 (1984).

107. Griffiths, P. D. et al. Cytomegalovirus glycoprotein-B vaccine with MF59 adjuvant in transplant recipients a phase 2 randomised placebo-controlled trial. Lancet 377, 1256-1263 (2011).

108. Baraniak, I. et al. Epitope-specific humoral responses to human cytomegalovirus glycoprotein-B vaccine with MF59: anti-AD2 levels correlate with protection from viremia. J. Infect. Dis. 217, 1907-1917 (2018).

109. Burke H. C. \& Heldwein, E. E. Crystal structure of the human cytomegalovirus glycoprotein B. PLoS Pathog. 11, e 1005227 (2015)

110. Plotkin, S. A. Complex correlates of protection after vaccination. Clin. Infect. Dis. 56, 1458-1465 (2013).
111. Ishida, J. H. et al. Phase 2 randomized, double-blind, placebo-controlled trial of RG7667, a combination monoclonal antibody, for prevention of cytomegalovirus infection in high-risk kidney transplant recipients. Antimicrob. Agents Chemother. 61, e01794-16 (2017).

112. Baraniak, I. A Reeves, M. B. \& Griffiths, P. D. Criteria to define interruption of transmission of human cytomegalovirus from organ donor to recipient Rev. Med. Virol. 28, e1958 (2018)

113. Kharfan-Dabaja, M. A. et al. A novel therapeutic cytomegalovirus DNA vaccine in allogeneic haemopoietic stem-cell transplantation: a randomised, double-blind, placebo-controlled, phase 2 trial. Lancet Infect. Dis. 12, 290-299 (2012).

114. Schwendinger, M. et al. A randomized dose-escalating phase I trial of a replication-deficient lymphocytic choriomeningitis virus vector-based vaccine against human cytomegalovirus. J. Infect. Dis. https://doi.org/ 10.1093/infdis/jiaa121 (2020)

115. Liu, Y. et al. A replication-defective human cytomegalovirus vaccine elicits humoral immune responses analogous to those with natural infection. J. Virol. 93, e00747-19 (2019).

116. Griffiths, P. \& Hughes, B. Choice of study populations for vaccines. J. Infect. Dis. 221, S128-S134 (2020).

117. Vutien, P. et al. Association of donor and recipient cytomegalovirus serostatus on graft and patient survival in liver transplant recipients. Liver Transpl. https://doi.org/10.1002/lt.26045 (2021).

118. Martinez-Martin, N. et al. An unbiased screen for human cytomegalovirus identifies neuropilin- 2 as a central viral receptor. Cell 174, 1158-1171.e19 (2018).

119. Murray, M. J., Peters, N. E. \& Reeves, M. B. Navigating the Host Cell Response during Entry into Sites of Latent Cytomegalovirus Infection. Pathogens 7, 30 (2018).

120. Nichols, W. G., Price, T. H., Gooley, T., Corey, L. \& Boeckh, M. Transfusion-transmitted cytomegalovirus infection after receipt of leukoreduced blood products. Blood 101, 4195-4200 (2003).

121. Ljungman, P., Hakki, M. \& Boeckh, M. Cytomegalovirus in hematopoietic stem cell transplant recipients. Hematol. Oncol. Clin. North Am. 25, 151-169 (2011).

122. Spector, S. A., Wong, R., Hsia, K., Pilcher, M. \& Stempien, M. J. Plasma cytomegalovirus (CMV) DNA load predicts CMV disease and survival in AIDS patients. J. Clin. Invest 101, 497-502 (1998).

123. Bowen, E. F. et al. Cytomegalovirus polymerase chain reaction viraemia in patients receiving ganciclovir maintenance therapy for retinitis. AIDS 12, 605-611 (1998).

124. Paya, C. et al. Efficacy and safety of valganciclovir vs. oral ganciclovir for prevention of cytomegalovirus disease in solid organ transplant recipients. $\mathrm{Am}$. J. Transplant. 4, 611-620 (2004)

125. Limaye, A. P., Corey, L., Koelle, D. M., Davis, C. L. \& Boeckh, M. Emergence of ganciclovir-resistant cytomegalovirus disease among recipients of solid-organ transplants. Lancet 356, 645-649 (2000).

126. Papanicolaou, G. A et al. Maribavir for refractory or resistant cytomegalovirus infections in hematopoieticcell or solid-organ transplant recipients: a randomized dose-ranging, double-blind, phase 2 study. Clin. Infect Dis. 68, 1255-1264 (2019).

127. Weller, T. H., Macauley, J. C., Craig, J. M. \& Wirth, P. Isolation of intranuclear inclusion producing agents from infants with illnesses resembling cytomegalic inclusion disease. Proc. Soc. Exp. Biol. Med. 94, 4-12 (1957).

128. Weller, T. H. The cytomegaloviruses: ubiquitous agents with protean clinical manifestations. I. N. Engl. J. Med. 285, 203-214 (1971).

129. Weller, T. H. The cytomegaloviruses: ubiquitous agents with protean clinical manifestations. II. N. Engl. J. Med. 285, 267-274 (1971).

130. Cha, T. A. et al. Human cytomegalovirus clinical isolates carry at least 19 genes not found in laboratory strains. J.Virol. 70, 78-83 (1996). This article is the first description of some laboratory-adapted HCMV strains having large deletions.

131. Revello, M. G. \& Gerna, G. Human cytomegalovirus tropism for endothelial/epithelial cells: scientific background and clinical implications. Rev. Med. Virol. 20, 136-155 (2010)

132. Nguyen, C. C. \& Kamil, J. P. Pathogen at the gates: human cytomegalovirus entry and cell tropism. Viruses 10, 704 (2018)

133. Wang, D. \& Shenk, T. Human cytomegalovirus virion protein complex required for epithelial and endothelial cell tropism. Proc. Natl Acad Sci. USA 102, 18153-18158 (2005).

134. Vanarsdall, A. L. et al. CD147 promotes entry of pentamer-expressing human cytomegalovirus into epithelial and endothelial cells. mBio 9, e00781-18 (2018).

135. E, X. et al. OR $14 \mathrm{I} 1$ is a receptor for the human cytomegalovirus pentameric complex and defines vira epithelial cell tropism. Proc. Natl Acad. Sci. USA 116 7043-7052 (2019).

136. Stanton, R. J. et al. Reconstruction of the complete human cytomegalovirus genome in a BAC reveals RL13 to be a potent inhibitor of replication. J. Clin. Invest. 120, 3191-3208 (2010)

This article shows that cloning of HCMV strain Merlin as a bacterial artificial chromosome allows simple manipulation of the genome and preservation without the selective pressures caused by propagation in cell cultures

137. Sijmons, S. et al. High-throughput analysis of human cytomegalovirus genome diversity highlights the widespread occurrence of gene-disrupting mutations and pervasive recombination. J. Virol. 89, 7673-7695 (2015).

This article shows that next-generation sequencing allows the full extent of genome variability to be documented in circulating strains.

138. Suarez, N. M. et al. Human cytomegalovirus genomes sequenced directly from clinical material: variation, multiple-strain infection, recombination, and gene loss. J. Infect. Dis. 220, 781-791 (2019).

This article applies next-generation sequencing to strains found directly in clinical material.

139. Lassalle, F. et al. Islands of linkage in an ocean of pervasive recombination reveals two-speed evolution of human cytomegalovirus genomes. Virus Evol. 2, vew017 (2016).

140. Dolan, A. et al. Genetic content of wild-type human cytomegalovirus. J. Gen. Virol. 85, 1301-1312 (2004).

141. Stern-Ginossar, N. et al. Decoding human cytomegalovirus. Science 338, 1088-1093 (2012).

142. Dunn, W. et al. Functional profiling of a human cytomegalovirus genome. Proc. Natl Acad. Sci. USA 100, 14223-14228 (2003)

143. Chou, S. W. \& Dennison, K. M. Analysis of interstrain variation in cytomegalovirus glycoprotein $B$ sequences encoding neutralization-related epitopes. J. Infect. Dis. 163, 1229-1234 (1991)

144. Nelson, C. S. et al. Intrahost dynamics of human cytomegalovirus variants acquired by seronegative glycoprotein B vaccinees. J. Virol. 93, e01695-18 (2019).

145. Wloch, M. K. et al. Safety and immunogenicity of a bivalent cytomegalovirus DNA vaccine in healthy adult subjects. J. Infect. Dis. 197, 1634-1642 (2008).

146. Hage, E. et al. Characterization of human cytomegalovirus genome diversity in immunocompromised hosts by whole-genome sequencing directly from clinical specimens. J. Infect. Dis. 215, 1673-1683 (2017).

147. Renzette, N. et al. Limits and patterns of cytomegalovirus genomic diversity in humans. Proc. Natl Acad. Sci. USA 112, E4120-E4128 (2015).

148. Suarez, N. M. et al. Whole-genome approach to assessing human cytomegalovirus dynamics in transplant patients undergoing antiviral therapy. Front. Cell Infect. Microbiol. 10, 267 (2020).

149. Quinnan, G. V. Jr. et al. Cytotoxic T cells in cytomegalovirus infection: HLA-restricted T-lymphocyte and non-T-lymphocyte cytotoxic responses correlate with recovery from cytomegalovirus infection in bonemarrow-transplant recipients. N. Engl. J. Med. 307 7-13 (1982).

150. Reusser, P., Riddell, S. R., Meyers, J. D. \& Greenberg, P. D. Cytotoxic T lymphocyte response to cytomegalovirus after human allogeneic bone marrow transplantation: pattern of recovery and correlation with cytomegalovirus infection and disease. Blood 78, 1373-1380 (1991)

151. Lilleri, D. et al. Human cytomegalovirus-specific $\mathrm{CD}^{+}$and $\mathrm{CD} 8{ }^{+} \mathrm{T}$-cell reconstitution in adult allogeneic hematopoietic stem cell transplant recipients and immune control of viral infection. Haematologica 93 , 248-256 (2008)

152. Gabanti, E. et al. Human cytomegalovirus (HCMV)specific $\mathrm{CD}^{+}$and $\mathrm{CD} 8^{+} \mathrm{T}$ cells are both required for prevention of HCMV disease in seropositive solidorgan transplant recipients. PLOS ONE 9, e106044 (2014).

153. Feuchtinger, T. et al. Adoptive transfer of pp65-specific $T$ cells for the treatment of chemorefractory 
cytomegalovirus disease or reactivation after haploidentical and matched unrelated stem cell transplantation. Blood 116, 4360-4367 (2010).

154. Kaeuferle, T., Krauss, R., Blaeschke, F., Willier, S. ¿ Feuchtinger, T. Strategies of adoptive T-cell transfer

to treat refractory viral infections post allogeneic

stem cell transplantation. J. Hematol. Oncol. 12, 13 (2019).

\section{Acknowledgements}

Work in the authors' laboratory is funded by the Wellcome Trust (WT/204870/Z/16/Z), the Medical Research Council (MRC) (MR/RO21384/1) and the National Institute for Health Research (NIHR) (II-LB-1117-20001)
Author contributions

Both authors researched data for the article. P.G. wrote the first draft, which was revised by M.R. Both authors contributed substantially to the discussion of content, reviewed the text and edited to form the final manuscript.

\section{Competing interests}

Both authors are co-inventors (along with I. Baraniak) on UK patent application number 2020135.6 assigned to University College London (UCL), entitled 'hCMV antibody and vaccine target', that deals with a novel antigenic domain on HCMV glycoprotein B (gB). UCL received funds from Taked pharmaceuticals to compensate for the time P.G. spent as a member of the end-point committee for a randomized clinical trial (RCT) of maribavir. The authors declare no other competing interests.

\section{Peer review information}

Nature Reviews Microbiology thanks N. Lemmermann who co-reviewed with S. Becker, C. Naucler, R. Stanton and the other, anonymous, reviewer(s) for their contribution to the peer review of this work.

\section{Publisher's note}

Springer Nature remains neutral with regard to jurisdictional claims in published maps and institutional affiliations.

(c) Springer Nature Limited 2021 